\title{
THE TOPOLOGICAL ASYMPTOTIC FOR THE NAVIER-STOKES EQUATIONS
}

\author{
SAmuel Amstutz ${ }^{1}$
}

\begin{abstract}
The aim of the topological asymptotic analysis is to provide an asymptotic expansion of a shape functional with respect to the size of a small inclusion inserted inside the domain. The main field of application is shape optimization. This paper addresses the case of the steady-state NavierStokes equations for an incompressible fluid and a no-slip condition prescribed on the boundary of an arbitrary shaped obstacle. The two and three dimensional cases are treated for several examples of cost functional and a numerical application is presented.
\end{abstract}

Mathematics Subject Classification. 35J60, 49Q10, 49Q12, 76D05, 76D55.

Received January 14, 2004. Revised July 19, 2004.

\section{INTRODUCTION}

Most topology optimization methods are based on the computation of the first variation of a shape functional with respect to a "small" perturbation of the physical properties of the material constitutive of the domain. This perturbation can be

- either of small amplitude and fixed support;

- or of narrow support with fixed amplitude.

In the frequent case where only two types of material are allowed, the first solution requires a relaxation of the problem. A rigorous and efficient way of doing this is provided by the homogenization theory $[1-3,5,14]$.

The topological sensitivity analysis is part of the second category. To present the basic idea, let us consider a shape functional $\mathcal{J}(\Omega)=J_{\Omega}\left(u_{\Omega}\right)$ where $u_{\Omega}$ is the solution of a given PDE defined in $\Omega \subset \mathbb{R}^{n}, n=2$ or 3 . For a small parameter $\rho>0$, the perturbed domain $\Omega_{\rho}$ is obtained by the creation of a small hole inside the initial domain $\Omega_{0}$, namely $\Omega_{\rho}=\Omega_{0} \backslash\left(\overline{x_{0}+\rho \omega}\right)$ where $\omega$ is a fixed and bounded subset of $\mathbb{R}^{n}$ containing the origin and $x_{0}$ is some point of $\Omega_{0}$. Generally, an asymptotic expansion can be written in the form

$$
\mathcal{J}\left(\Omega_{\rho}\right)-\mathcal{J}\left(\Omega_{0}\right)=f(\rho) g\left(x_{0}\right)+o(f(\rho))
$$

where $f$ is a positive function going to zero with $\rho$. This expansion is called the topological asymptotic and $g$ the topological gradient, or topological derivative. In order to minimize the criterion, the best location to create a small hole is the point where $g$ reaches its minimum. When the topological gradient is easy to compute, this remark leads to efficient shape optimization algorithms.

The topological sensitivity analysis was introduced by Schumacher [23], Sokolowski and Zochowski [25] for the compliance minimization in linear elasticity with a Neumann condition on the boundary of the hole.

\footnotetext{
Keywords and phrases. Shape optimization, topological asymptotic, Navier-Stokes equations.

1 Mathématiques pour l'Industrie et la Physique, UMR 5640, CNRS-Université Paul Sabatier-INSA, 118 route de Narbonne, 31062 Toulouse Cedex 4, France; amstutz@mip.ups-tlse.fr
}

(c) EDP Sciences, SMAI 2005 
Then, Masmoudi [15] worked out a topological sensitivity framework based on a generalization of the adjoint method and on the use of a truncation technique to give an equivalent formulation of the PDE in a fixed functional space. By using this approach, Garreau, Guillaume, Masmoudi and Sid Idris have obtained the topological asymptotic expression for the linear elasticity [9], the Poisson [10] and the Stokes [11] equations with an arbitrary shaped hole and a large class of shape functionals. The analysis is more difficult when the differential operator under consideration is non-homogeneous. For a Dirichlet condition prescribed on the hole, the Helmholtz [21,22] and the quasi-Stokes [12] problems have been treated. The case of a Neumann condition has also been addressed [4] with the help of an alternative to the truncation based on the comparison between the perturbed and the initial problems both formulated in the perforated domain. This approach brings in this case substantial technical simplifications. For completeness, we refer the reader to the publications $[7,13,16-20]$ where the asymptotic behavior of the solution in various situations is studied.

The present work deals with the steady Navier-Stokes equations for an incompressible fluid. The aim is to provide a tool for shape optimization in fluid dynamics, and this contribution can be seen as the first step towards the evolution and compressible case. From the mathematical point of view, the difficulties are raised by the nonlinearity of the operator. Here, the natural boundary condition on the hole (an obstacle in this context) is of Dirichlet type. An extension of the perturbed velocity field by zero inside the inclusion makes possible the use of an adjoint method in the whole domain, avoiding a truncation which would present some theoretical difficulties because of the nonlinearity. We show that the effect of the nonlinear term on the asymptotic behavior of the cost functional is of second order: we obtain the same formulas as for the Stokes problem [11]. We recall the results holding for a spherical obstacle in $3 \mathrm{D}$ and for an arbitrary shaped obstacle in $2 \mathrm{D}$, valid for a functional which does not involve the gradient of the velocity in the vicinity of the obstacle:

$$
\mathcal{J}\left(\Omega_{\rho}\right)-\mathcal{J}\left(\Omega_{0}\right)= \begin{cases}6 \pi \nu \rho u_{0}\left(x_{0}\right) \cdot v_{0}\left(x_{0}\right)+o(\rho) & \text { in } 3 \mathrm{D} \\ \frac{-4 \pi \nu}{\ln \rho} u_{0}\left(x_{0}\right) \cdot v_{0}\left(x_{0}\right)+o\left(\frac{-1}{\ln \rho}\right) & \text { in } 2 \mathrm{D}\end{cases}
$$

where $u_{0}$ and $v_{0}$ denote the direct and adjoint velocity fields, respectively. In particular, it appears that the function $f(\rho)$ is the same for all the Dirichlet problems that have been treated until now: $f(\rho)=\rho$ in 3D, $f(\rho)=-1 / \ln \rho$ in 2D. For comparison, we recall that it has been found $f(\rho)=\rho^{n}$ in the Neumann cases.

The precise assumptions on the problem are described in Section 2. In Section 3, we present the main results as well as the sketch of the asymptotic analysis. The 3D and 2D cases are studied separately. The proofs are gathered in Section 4. Section 5 is devoted to a numerical application.

\section{Presentation of the problem and notations}

\subsection{The Navier-Stokes problem in a perforated domain}

Let $\Omega$ be a bounded domain of $\mathbb{R}^{n}, n=2$ or 3 , containing a Newtonian and incompressible fluid with coefficient of kinematic viscosity $\nu>0$. For simplicity, and without any loss of generality, we assume that the system of units is chosen in such a way that the density is equal to one. The velocity and pressure fields are denoted by $u_{0}$ and $p_{u_{0}}$, respectively. For that fluid, the Navier-Stokes equations read:

$$
\left\{\begin{aligned}
-\nu \Delta u_{0}+\nabla u_{0} \cdot u_{0}+\nabla p_{u_{0}}=0 & \text { in } \Omega, \\
\operatorname{div} u_{0}=0 & \text { in } \Omega, \\
u_{0}=U & \text { on } \Gamma .
\end{aligned}\right.
$$

We use the standard notation $\nabla u \cdot v=\sum_{i=1}^{n} \partial_{i} u v_{i}, v_{i}$ and $\partial_{i}$ being the i-th component of the vector $v$ and the partial derivative with respect to the i-th coordinate, respectively. For well-posedness, we suppose that the boundary $\Gamma$ of $\Omega$ is smooth (see the precise conditions in [26] Appendix 1, p. 458), and that $U$, the prescribed 


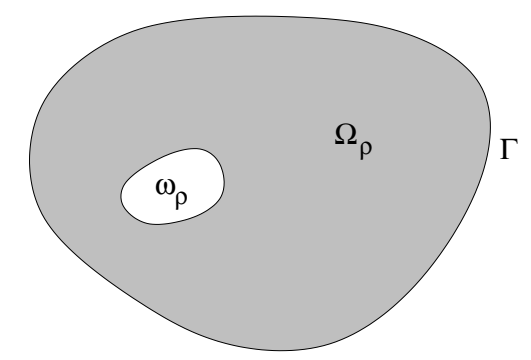

FiguRE 1. The perforated domain.

velocity on the boundary, belongs to the functional space $H^{3 / 2}(\Gamma)^{n}$ and satisfies the normalization condition: for all connected component $\Gamma_{i}$ of $\Gamma$,

$$
\int_{\Gamma_{i}} U \cdot \mathbf{n} \mathrm{d} s=0 .
$$

In all the paper, $\mathbf{n}$ stands for the outward unit normal of the boundary under consideration.

For a given $x_{0} \in \Omega$, we consider the perforated domain $\Omega_{\rho}=\Omega \backslash \omega_{\rho}, \omega_{\rho}=x_{0}+\rho \omega$, where $\omega$ is a fixed bounded domain of $\mathbb{R}^{n}$ containing the origin and whose boundary $\partial \omega$ is connected and of class $\mathcal{C}^{2}$ (see Fig. 1). Possibly shifting the origin, we suppose henceforth that $x_{0}=0$. The new velocity and pressure fields solve the system:

$$
\left\{\begin{aligned}
-\nu \Delta u_{\rho}+\nabla u_{\rho} \cdot u_{\rho}+\nabla p_{u_{\rho}}=0 & \text { in } \Omega_{\rho}, \\
\operatorname{div} u_{\rho}=0 & \text { in } \Omega_{\rho}, \\
u_{\rho}=U & \text { on } \Gamma, \\
u_{\rho}=0 & \text { on } \partial \omega_{\rho} .
\end{aligned}\right.
$$

\subsection{Variational formulation and well-posedness}

We define the family of functional spaces $\left(\mathcal{V}_{\rho}\right)_{\rho \geq 0}$ by

$$
\begin{gathered}
\mathcal{V}_{0}=\left\{u \in H^{1}(\Omega)^{n}, \operatorname{div} u=0\right\}, \\
\mathcal{V}_{\rho}=\left\{u \in H^{1}\left(\Omega_{\rho}\right)^{n}, \operatorname{div} u=0, u_{\mid \partial \omega_{\rho}}=0\right\} \quad \forall \rho>0 .
\end{gathered}
$$

We associate to Problem (2.3) the variational formulation: find $u_{\rho} \in \mathcal{V}_{\rho}$ such that $u_{\rho \mid \Gamma}=U$ and

$$
\int_{\Omega_{\rho}}\left[\nu \nabla u_{\rho}: \nabla \varphi+\left(\nabla u_{\rho} \cdot u_{\rho}\right) \cdot \varphi\right] \mathrm{d} x=0 \quad \forall \varphi \in \mathcal{V}_{\rho}, \varphi_{\mid \Gamma}=0
$$

We remark that the pressure does not appear here. It can be deduced afterwards from the velocity field, up to an additive constant. This is a well-known phenomenon for incompressible flows. In [26] (Appendix 1, p. 469), it is proved that Problem (2.4) has at least one solution. Henceforth, all Navier-Stokes equations have to be understood in the sense of their variational formulations. We assume that

$$
\left|u_{0}\right|_{1, \Omega}<\frac{\nu}{k}
$$

where the constant $k$ is defined by

$$
k= \begin{cases}\frac{2 \sqrt{2}}{3} \operatorname{meas}(\Omega)^{1 / 6} & \text { if } n=3 \\ \frac{1}{2} \operatorname{meas}(\Omega)^{1 / 2} & \text { if } n=2 .\end{cases}
$$


In [8] (Vol. 2, Chap. VIII, p. 10), it is proved that (2.5) is a sufficient uniqueness condition for Problem (2.1). Thanks to estimate (4.6) which is stated and proved in Section 4, we obtain that (2.5) implies $\left|u_{\rho}\right|_{1, \Omega_{\rho}}<\nu / k$ for any $\rho$ sufficiently small and thus, for such a $\rho$, Problem (2.3) admits a unique solution.

\subsection{The cost functional}

We are interested in a criterion of the form

$$
j(\rho)=J_{\rho}\left(u_{\rho}\right)
$$

where $J_{\rho}$ is a differentiable function from $\mathcal{V}_{\rho}$ into $\mathbb{R}$. For simplicity, the analysis is restricted to the cases where $J_{\rho}$ belongs to one of the following categories.

Case 1. There exists a neighborhood $\mathcal{O}$ of the origin and a differentiable map $J:\left\{u \in H^{1}(\Omega \backslash \overline{\mathcal{O}})^{n}\right.$, div $u=$ $0\} \rightarrow \mathbb{R}$ such that for all $u \in \mathcal{V}_{\rho}$

$$
J_{\rho}(u)=J\left(u_{\mid \Omega \backslash \overline{\mathcal{O}}}\right) .
$$

Moreover, the distribution $D J_{0}\left(u_{0}\right)$ belongs to $H^{1}(\Omega)^{n}$.

Case 2. The cost functional is of the form

$$
J_{\rho}(u)=\int_{\Omega_{\rho}}\left|u-u_{d}\right|^{2} \mathrm{~d} x
$$

where $u_{d} \in H^{1}(\Omega)^{n}$.

Case 3. The cost functional is of the form

$$
J_{\rho}(u)=\nu \int_{\Omega_{\rho}}\left|\nabla u-\nabla u_{d}\right|^{2} \mathrm{~d} x
$$

where $u_{d} \in H^{2}(\Omega)^{n}$.

\subsection{Reformulation in a fixed functional space}

Equation (2.4) is written for a test function belonging to the variable functional space $\mathcal{V}_{\rho}$. This does not suit to the use of an adjoint method, which is however appropriate in our case since the criteria of interest are scalar. For this reason, we are going to reformulate equation (2.4) with respect to the fixed space $\mathcal{V}_{0}$.

Let us first define the map

$$
\begin{aligned}
F_{0}: \mathcal{V}_{0} & \longrightarrow \mathcal{V}_{0}^{\prime} \\
\left\langle F_{0}(u), \varphi\right\rangle & =\int_{\Omega}\left[\nu \nabla u_{\rho}: \nabla \varphi+\left(\nabla u_{\rho} \cdot u_{\rho}\right) \cdot \varphi\right] \mathrm{d} x \quad \forall u, \varphi \in \mathcal{V}_{0} .
\end{aligned}
$$

In this way, the variational formulation of (2.1) reads

$$
\left\langle F_{0}\left(u_{0}\right), \varphi\right\rangle=0 \quad \forall \varphi \in \mathcal{V}_{0}, \varphi_{\mid \Gamma}=0 .
$$

Let us now consider a parameter $\rho>0$. The extension by zero in $\omega_{\rho}$ defines an embedding from $\mathcal{V}_{\rho}$ into $\mathcal{V}_{0}$. This embedding will be considered in all the paper as "canonical": the extension by zero of a function $u \in \mathcal{V}_{\rho}$ will be still denoted by $u$. Thanks to a regularity property up to the boundary (see [8], Chap. VIII, p. 48), we have that $\left(u_{\rho}, p_{u_{\rho}}\right)$ is $H^{2} \times H^{1}$ in the vicinity of $\partial \omega_{\rho}$. Therefore, we can construct the map:

$$
\begin{gathered}
F_{\rho}: \mathcal{V}_{0} \longrightarrow \mathcal{V}_{0}^{\prime} \\
\left\langle F_{\rho}(u), \varphi\right\rangle=\left\langle F_{0}(u), \varphi\right\rangle+\int_{\partial \omega_{\rho}}\left(\nu \partial_{n} u_{\rho}-p_{u_{\rho}} \mathbf{n}\right) \cdot \varphi \mathrm{d} s \quad \forall u, \varphi \in \mathcal{V}_{0}
\end{gathered}
$$


We derive from the Green formula the equality:

$$
\left\langle F_{\rho}\left(u_{\rho}\right), \varphi\right\rangle=0 \quad \forall \varphi \in \mathcal{V}_{0}, \varphi_{\mid \Gamma}=0
$$

\subsection{The adjoint problem}

It is standard in control theory to define the adjoint state $v_{0}$ as the solution of the linearized problem: find $v_{0} \in \mathcal{V}_{0}, v_{0 \mid \Gamma}=0$ such that

$$
\left\langle D F_{0}\left(u_{0}\right) \varphi, v_{0}\right\rangle=-D J_{0}\left(u_{0}\right) \varphi \quad \forall \varphi \in \mathcal{V}_{0}
$$

Yet, we have for all $u, \varphi, v \in \mathcal{V}_{0}$ :

$$
\left\langle D F_{0}(u) \varphi, v\right\rangle=\int_{\Omega}[\nu \nabla \varphi: \nabla v+(\nabla \varphi \cdot u+\nabla u \cdot \varphi) \cdot v] \mathrm{d} x .
$$

If moreover $\varphi_{\mid \Gamma}=0$, then an integration by parts yields

$$
\left\langle D F_{0}(u) \varphi, v\right\rangle=\int_{\Omega}\left[\nu \nabla v: \nabla \varphi+\left(-\nabla v \cdot u+\nabla u^{T} \cdot v\right) \cdot \varphi\right] \mathrm{d} x .
$$

Therefore, the classical formulation associated to Problem $(2.11)$ reads: find $\left(v_{0}, p_{v_{0}}\right) \in H^{1}(\Omega)^{n} \times L^{2}(\Omega)$ such that

$$
\left\{\begin{aligned}
-\nu \Delta v_{0}-\nabla v_{0} \cdot u_{0}+\nabla u_{0}^{T} \cdot v_{0}+\nabla p_{v_{0}} & =-D J_{0}\left(u_{0}\right) & & \text { in } \Omega, \\
\operatorname{div} v_{0} & =0 & & \text { in } \Omega, \\
v_{0} & =0 & & \text { on } \Gamma .
\end{aligned}\right.
$$

Since $u_{0}$ satisfies (2.5), the existence and uniqueness of the solution of (2.12) is a consequence of the Lax-Milgram theorem. Indeed, the coercivity of the associated bilinear form comes from Lemmas 4.1 and 4.2 : for all $v \in H^{1}(\Omega)^{n}$,

$$
\begin{aligned}
\left|\left\langle D F_{0}\left(u_{0}\right) v, v\right\rangle_{\mathcal{V}_{0}^{\prime}, \mathcal{V}_{0}}\right| & =\left|\int_{\Omega}\left[\nu|\nabla v|^{2}+\left(\nabla u_{0} \cdot v\right) \cdot v\right] \mathrm{d} x\right| \\
& \geq \nu|v|_{1, \Omega}^{2}-k\left|u_{0}\right|_{1, \Omega}|v|_{1, \Omega}^{2} \\
& \geq \alpha|v|_{1, \Omega}^{2}, \quad \text { with } \alpha=\nu-k\left|u_{0}\right|_{1, \Omega}>0 .
\end{aligned}
$$

\section{Asymptotic Behavior of the Cost functional}

\subsection{A preliminary proposition}

The following proposition describes the adjoint method that will be used to calculate the first variation of the cost functional. The notations are those introduced in Section 2.

Proposition 3.1. If we determine asymptotic expansions of the form

$$
\begin{aligned}
\left\langle F_{\rho}\left(u_{\rho}\right)-F_{0}\left(u_{0}\right)-D F_{0}\left(u_{0}\right)\left(u_{\rho}-u_{0}\right), v_{0}\right\rangle & =f(\rho) \delta_{F}+o(f(\rho)), \\
J_{\rho}\left(u_{\rho}\right)-J_{0}\left(u_{0}\right)-D J_{0}\left(u_{0}\right)\left(u_{\rho}-u_{0}\right) & =f(\rho) \delta_{J}+o(f(\rho)),
\end{aligned}
$$

where $\delta_{F}, \delta_{J} \in \mathbb{R}$ and $f$ is a smooth function tending to zero with $\rho$, then

$$
j(\rho)-j(0)=f(\rho)\left(\delta_{F}+\delta_{J}\right)+o(f(\rho)) .
$$

Proof. By equations (2.8) and (2.10), we have

$$
j(\rho)-j(0)=J_{\rho}\left(u_{\rho}\right)-J_{0}\left(u_{0}\right)+\left\langle F_{\rho}\left(u_{\rho}\right)-F_{0}\left(u_{0}\right), v_{0}\right\rangle .
$$


Next, equations (3.1) and (3.2) yield

$$
j(\rho)-j(0)=D J_{0}\left(u_{0}\right)\left(u_{\rho}-u_{0}\right)+f(\rho) \delta_{J}+o(f(\rho))+\left\langle D F_{0}\left(u_{0}\right)\left(u_{\rho}-u_{0}\right), v_{0}\right\rangle+f(\rho) \delta_{F}+o(f(\rho)) .
$$

From equation (2.11) we derive the desired result.

We are now in position to carry out the topological asymptotic analysis. The 3D and 2D cases will be studied separately. The main steps will be

(1) to study the asymptotic behavior of the solution for the norms needed;

(2) to determine $f(\rho)$ and $\delta_{F}$ such that equation (3.1) holds;

(3) to check equation (3.2) for the cost functionals of interest.

\subsection{Asymptotic analysis in $3 \mathrm{D}$}

\subsubsection{Asymptotic behavior of the solution}

We are going to determine the leading term of the variation of the solution by two successive approximations. The error estimates (for the appropriate norms) are reported in Section 4.3.

- First approximation. We split $\left(u_{\rho}, p_{u_{\rho}}\right)$ into

$$
\left(u_{\rho}, p_{u_{\rho}}\right)=\left(u_{0}, p_{u_{0}}\right)+\left(h_{\rho}, p_{h_{\rho}}\right)+\left(r_{\rho}, p_{r_{\rho}}\right)
$$

where $\left(h_{\rho}, p_{h_{\rho}}\right)$ and $\left(r_{\rho}, p_{r_{\rho}}\right)$ solve the systems:

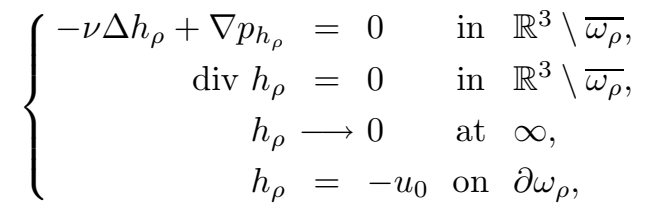

$$
\begin{aligned}
& \left\{\begin{aligned}
-\nu \Delta r_{\rho} & +\nabla r_{\rho} \cdot\left(u_{0}+h_{\rho}\right)+\nabla\left(u_{0}+h_{\rho}\right) \cdot r_{\rho}+\nabla r_{\rho} \cdot r_{\rho}+\nabla p_{r_{\rho}} & & \\
& =-\nabla h_{\rho} \cdot u_{0}-\nabla u_{0} \cdot h_{\rho}-\nabla h_{\rho} \cdot h_{\rho} & & \text { in } \Omega_{\rho}, \\
\operatorname{div} r_{\rho} & =0 & & \text { in } \Omega_{\rho}, \\
r_{\rho} & =-h_{\rho} & & \text { on } \Gamma, \\
r_{\rho} & =0 & & \text { on } \partial \omega_{\rho} .
\end{aligned}\right.
\end{aligned}
$$

It is proved in [6] (Chap. XI, p. 695) that the Stokes problem (3.3) has one and only one solution $\left(h_{\rho}, p_{h_{\rho}}\right) \in W^{1}\left(\mathbb{R}^{3} \backslash \overline{\omega_{\rho}}\right)^{3} \times L^{2}\left(\mathbb{R}^{3} \backslash \overline{\omega_{\rho}}\right) / \mathbb{R}$. We recall that, for any open and bounded subset $\mathcal{O}$ of $\mathbb{R}^{3}$, the space $W^{1}\left(\mathbb{R}^{3} \backslash \overline{\mathcal{O}}\right)$ is defined by (see [6], Chap. XI, p. 649)

$$
W^{1}\left(\mathbb{R}^{3} \backslash \overline{\mathcal{O}}\right)=\left\{u, \frac{u}{\left(1+r^{2}\right)^{1 / 2}} \in L^{2}\left(\mathbb{R}^{3} \backslash \overline{\mathcal{O}}\right), \nabla u \in L^{2}\left(\mathbb{R}^{3} \backslash \overline{\mathcal{O}}\right)^{3}\right\} .
$$

By difference, the existence of a solution of (3.4) is guaranteed.

- Second approximation. We set $H_{\rho}(x)=h_{\rho}(\rho x)$ and $P_{H_{\rho}}(x)=\rho p_{h_{\rho}}(x)$. We have

$$
\left\{\begin{aligned}
-\nu \Delta H_{\rho}+\nabla P_{H_{\rho}} & =0 & & \text { in } \mathbb{R}^{3} \backslash \bar{\omega}, \\
\operatorname{div} H_{\rho} & =0 & & \text { in } \mathbb{R}^{3} \backslash \bar{\omega}, \\
H_{\rho} & \longrightarrow 0 & & \text { at } \infty, \\
H_{\rho} & =-u_{0}(\rho x) & & \text { on } \partial \omega .
\end{aligned}\right.
$$


Then, we split $\left(H_{\rho}, P_{H_{\rho}}\right)$ into

$$
\left(H_{\rho}, P_{H_{\rho}}\right)=\left(H, P_{H}\right)+\left(S_{\rho}, P_{S_{\rho}}\right)
$$

where $\left(H, P_{H}\right)$ and $\left(S_{\rho}, P_{S_{\rho}}\right)$ are the solutions of:

$$
\begin{aligned}
& \left\{\begin{aligned}
-\nu \Delta H+\nabla P_{H} & =0 & & \text { in } \mathbb{R}^{3} \backslash \bar{\omega} \\
\operatorname{div} H & =0 & & \text { in } \mathbb{R}^{3} \backslash \bar{\omega} \\
H & \longrightarrow 0 & & \text { at } \infty \\
H & =-u_{0}(0) & & \text { on } \partial \omega
\end{aligned}\right. \\
& \left\{\begin{aligned}
-\nu \Delta S_{\rho}+\nabla P_{S_{\rho}} & =0 & & \text { in } \mathbb{R}^{3} \backslash \bar{\omega}, \\
\operatorname{div} S_{\rho} & =0 & & \text { in } \mathbb{R}^{3} \backslash \bar{\omega}, \\
S_{\rho} & \longrightarrow 0 & & \text { at } \infty, \\
S_{\rho} & =-u_{0}(\rho x)+u_{0}(0) & & \text { on } \partial \omega .
\end{aligned}\right.
\end{aligned}
$$

Problem (3.6) can be solved with the help of a single layer potential (see [6], Chap. XI, p. 697):

$$
\left\{\begin{array}{l}
H(x)=\int_{\partial \omega} E(x-y) \eta(y) \mathrm{d} s(y) \\
P_{H}(x)=\int_{\partial \omega} \Pi(x-y) \cdot \eta(y) \mathrm{d} s(y)
\end{array}\right.
$$

where $\eta \in H^{-1 / 2}(\partial \omega)^{3}$ verifies the integral equation

$$
\int_{\partial \omega} E(x-y) \eta(y) \mathrm{d} s(y)=-u_{0}(0) \quad \forall x \in \partial \omega
$$

and $(E, \Pi)$ is the fundamental solution of the Stokes system

$$
E(x)=\frac{1}{8 \pi \nu|x|}\left(I+\mathbf{e}_{\mathbf{r}} \mathbf{e}_{\mathbf{r}}^{T}\right), \quad \Pi(x)=\frac{\mathbf{e}_{\mathbf{r}}}{4 \pi|x|^{2}} .
$$

We denote by $|x|$ the euclidean norm of the vector $x$ and by $\mathbf{e}_{\mathbf{r}}$ the unit vector $\mathbf{e}_{\mathbf{r}}=x /|x|$. The density $\eta$ is unique up to a function proportional to the normal. As we will see, for some particular norms, the pair

$$
\left(H\left(\frac{x}{\rho}\right), \frac{1}{\rho} P_{H}\left(\frac{x}{\rho}\right)\right)
$$

is the leading term of the variation $\left(u_{\rho}-u_{0}, p_{u_{\rho}}-p_{u_{0}}\right)$ in $\Omega_{\rho}$.

\subsubsection{Asymptotic behavior of the cost functional}

Our aim here is to find some parameters $f(\rho)$ and $\delta_{F}$ such that equation (3.1) holds. Hence we have to study the quantity

$$
\begin{aligned}
V_{F}(\rho) & :=\left\langle F_{\rho}\left(u_{\rho}\right)-F_{0}\left(u_{0}\right)-D F_{0}\left(u_{0}\right)\left(u_{\rho}-u_{0}\right), v_{0}\right\rangle \\
& =\left\langle F_{\rho}\left(u_{\rho}\right)-F_{0}\left(u_{\rho}\right), v_{0}\right\rangle+\left\langle F_{\rho}\left(u_{\rho}\right)-F_{0}\left(u_{0}\right)-D F_{0}\left(u_{0}\right)\left(u_{\rho}-u_{0}\right), v_{0}\right\rangle \\
& =\int_{\partial \omega_{\rho}}\left(\nu \partial_{n} u_{\rho}-p_{u_{\rho}} \mathbf{n}\right) \cdot v_{0} \mathrm{~d} s+\int_{\Omega}\left(\nabla\left(u_{\rho}-u_{0}\right) \cdot\left(u_{\rho}-u_{0}\right)\right) \cdot v_{0} \mathrm{~d} x .
\end{aligned}
$$


Denoting

we obtain successively

$$
\begin{gathered}
\mathcal{E}_{1}(\rho)=\int_{\Omega}\left(\nabla\left(u_{\rho}-u_{0}\right) \cdot\left(u_{\rho}-u_{0}\right)\right) \cdot v_{0} \mathrm{~d} x \\
\mathcal{E}_{2}(\rho)=\int_{\partial \omega_{\rho}}\left(\nu \partial_{n} u_{0}-p_{u_{0}} \mathbf{n}\right) \cdot v_{0} \mathrm{~d} s \\
\mathcal{E}_{3}(\rho)=\int_{\partial \omega_{\rho}}\left(\nu \partial_{n} r_{\rho}-p_{r_{\rho}} \mathbf{n}\right) \cdot v_{0} \mathrm{~d} s \\
\mathcal{E}_{4}(\rho)=\rho \int_{\partial \omega}\left(\nu \partial_{n} S_{\rho}-P_{S_{\rho}} \mathbf{n}\right) \cdot v_{0}(\rho x) \mathrm{d} s
\end{gathered}
$$

$$
\begin{aligned}
V_{F}(\rho) & =\int_{\partial \omega_{\rho}}\left(\nu \partial_{n} h_{\rho}-p_{h_{\rho}} \mathbf{n}\right) \cdot v_{0} \mathrm{~d} s+\sum_{i=1}^{3} \mathcal{E}_{i}(\rho) \\
& =\rho \int_{\partial \omega}\left(\nu \partial_{n} H_{\rho}-P_{H_{\rho}} \mathbf{n}\right) \cdot v_{0}(\rho x) \mathrm{d} s+\sum_{i=1}^{3} \mathcal{E}_{i}(\rho) \\
& =\rho \int_{\partial \omega}\left(\nu \partial_{n} H-P_{H} \mathbf{n}\right) \cdot v_{0}(\rho x) \mathrm{d} s+\sum_{i=1}^{4} \mathcal{E}_{i}(\rho) .
\end{aligned}
$$

Then, the jump relation of the single layer potential yields (see [6] p. 698)

$$
\begin{aligned}
V_{F}(\rho) & =-\rho \int_{\partial \omega} \eta \cdot v_{0}(\rho x) \mathrm{d} s+\sum_{i=1}^{4} \mathcal{E}_{i}(\rho) \\
& =-\rho\left(\int_{\partial \omega} \eta \mathrm{d} s\right) \cdot v_{0}(0)+\sum_{i=1}^{5} \mathcal{E}_{i}(\rho),
\end{aligned}
$$

where

$$
\mathcal{E}_{5}(\rho)=-\rho \int_{\partial \omega} \eta \cdot\left[v_{0}(\rho x)-v_{0}(0)\right] \mathrm{d} s .
$$

It is convenient to introduce the polarization matrix $\mathcal{P}_{\omega}$ defined by

$$
\mathcal{P}_{\omega}=\int_{\partial \omega} \Lambda(x) \mathrm{d} s(x),
$$

where $\Lambda$ is the 3 by 3 matrix solution of the integral equation

$$
\int_{\partial \omega} E(x-y) \Lambda(y) \mathrm{d} s(y)=I \quad \forall x \in \partial \omega .
$$

We denote here by $I$ the identity matrix of order 3 . Then we can write

$$
V_{F}(\rho)=\rho \mathcal{P}_{\omega} u_{0}(0) \cdot v_{0}(0)+\sum_{i=1}^{5} \mathcal{E}_{i}(\rho) .
$$

We will prove in Section 4 that $\left|\mathcal{E}_{i}(\rho)\right|=o(\rho)$ for all $i=1, \ldots, 5$. Thus, it stands out that equation (3.1) holds with

$$
f(\rho)=\rho \quad \text { and } \quad \delta_{F}=\mathcal{P}_{\omega} u_{0}(0) \cdot v_{0}(0) .
$$


3.2.3. Polarization matrix for a spherical hole

Consider the most natural case where $\omega=B(0,1)$, the unit ball of $\mathbb{R}^{3}$. We have in this particular situation

$$
\int_{\partial \omega} E(x-y) \mathrm{d} s=\frac{2}{3 \nu} I \quad \forall x \in \partial \omega .
$$

Hence, the solution of (3.10) reads

$$
\Lambda(y)=\frac{3 \nu}{2} I \quad \forall y \in \partial \omega
$$

It follows the polarization matrix

$$
\mathcal{P}_{B(0,1)}=6 \pi \nu I
$$

\subsection{Asymptotic analysis in $\mathbf{2 D}$}

\subsubsection{Asymptotic behavior of the solution}

In dimension two, the fundamental solution of the Stokes problem reads

$$
E(x)=\frac{1}{4 \pi \nu}\left(-\ln |x| I+\mathbf{e}_{\mathbf{r}} \mathbf{e}_{\mathbf{r}}^{T}\right), \quad \Pi(x)=\frac{\mathbf{e}_{\mathbf{r}}}{2 \pi|x|} .
$$

The matrix $E(x)$ does not tend to zero at infinity. That is why the argumentation is very different from what was done in $3 \mathrm{D}$.

We split $\left(u_{\rho}, p_{u_{\rho}}\right)$ into

$$
\left(u_{\rho}, p_{u_{\rho}}\right)=\left(u_{0}, p_{u_{0}}\right)+\left(h_{\rho}, p_{h_{\rho}}\right)+\left(r_{\rho}, p_{r_{\rho}}\right)
$$

with:

$$
\begin{aligned}
& \left\{\begin{array}{l}
h_{\rho}=\frac{-1}{\ln \rho}\left(H-H_{\rho}\right) \\
p_{h_{\rho}}(x)=\frac{-1}{\ln \rho}\left(P_{H}-P_{H_{\rho}}\right)
\end{array}\right. \\
& \left\{\begin{array}{l}
H(x)=-4 \pi \nu E(x) u_{0}(0), \\
P_{H}(x)=-4 \pi \nu \Pi(x) \cdot u_{0}(0),
\end{array}\right. \\
& \left\{\begin{array}{l}
-\nu \Delta H_{\rho}+\nabla H_{\rho} \cdot\left(u_{0}-\frac{1}{\ln \rho} H\right)+\left(\nabla u_{0}-\frac{1}{\ln \rho} \nabla H\right) \cdot H_{\rho}+\frac{1}{\ln \rho} \nabla H_{\rho} \cdot H_{\rho}+\nabla P_{H_{\rho}} \\
=\nabla u_{0} \cdot H+\nabla H \cdot u_{0}-\frac{1}{\ln \rho} \nabla H \cdot H \text { in } \Omega, \\
\operatorname{div} H_{\rho}=0 \text { in } \Omega, \\
H_{\rho}=H \text { on } \Gamma,
\end{array}\right. \\
& \left\{\begin{array}{rlrl}
-\nu \Delta r_{\rho}+\nabla r_{\rho} \cdot\left(u_{0}+h_{\rho}\right)+\nabla\left(u_{0}+h_{\rho}\right) \cdot r_{\rho}+\nabla r_{\rho} \cdot r_{\rho}+\nabla p_{r_{\rho}} & =0 & & \text { in } \Omega_{\rho}, \\
\operatorname{div} r_{\rho} & =0 & \text { in } \Omega_{\rho}, \\
r_{\rho} & =0 & \text { on } \Gamma, \\
r_{\rho} & =-u_{0}-h_{\rho} & \text { on } \partial \omega_{\rho} .
\end{array}\right.
\end{aligned}
$$

The existence of solutions of problems of the type (3.13) and (3.14) is shown in [8] (Chap. VIII, p. 20). 
3.3.2. Asymptotic behavior of the cost functional

We consider again the variation

$$
\begin{aligned}
V_{F}(\rho) & :=\left\langle F_{\rho}\left(u_{\rho}\right)-F_{0}\left(u_{0}\right)-D F_{0}\left(u_{0}\right)\left(u_{\rho}-u_{0}\right), v_{0}\right\rangle \\
& =\int_{\partial \omega_{\rho}}\left(\nu \partial_{n} u_{\rho}-p_{u_{\rho}} \mathbf{n}\right) \cdot v_{0} \mathrm{~d} s+\int_{\Omega}\left(\nabla\left(u_{\rho}-u_{0}\right) \cdot\left(u_{\rho}-u_{0}\right)\right) \cdot v_{0} \mathrm{~d} x .
\end{aligned}
$$

The introduction of the errors

$$
\begin{gathered}
\mathcal{E}_{1}(\rho)=\int_{\Omega}\left(\nabla\left(u_{\rho}-u_{0}\right) \cdot\left(u_{\rho}-u_{0}\right)\right) \cdot v_{0} \mathrm{~d} x \\
\mathcal{E}_{2}(\rho)=\int_{\partial \omega_{\rho}}\left(\nu \partial_{n} u_{0}-p_{u_{0}} \mathbf{n}\right) \cdot v_{0} \mathrm{~d} s \\
\mathcal{E}_{3}(\rho)=\int_{\partial \omega_{\rho}}\left(\nu \partial_{n} r_{\rho}-p_{r_{\rho}} \mathbf{n}\right) \cdot v_{0} \mathrm{~d} s \\
\mathcal{E}_{4}(\rho)=\int_{\partial \omega_{\rho}}\left(\nu \partial_{n} h_{\rho}-p_{h_{\rho}} \mathbf{n}\right) \cdot\left(v_{0}-v_{0}(0)\right) \mathrm{d} s \\
\mathcal{E}_{5}(\rho)=\frac{-1}{\ln \rho} \int_{\partial \omega_{\rho}}\left(\nu \partial_{n} H_{\rho}-P_{H_{\rho}} \mathbf{n}\right) \cdot v_{0}(0) \mathrm{d} s
\end{gathered}
$$

permits to write

$$
\begin{aligned}
V_{F}(\rho) & =\int_{\partial \omega_{\rho}}\left(\nu \partial_{n} h_{\rho}-p_{h_{\rho}} \mathbf{n}\right) \cdot v_{0}(0) \mathrm{d} s+\sum_{i=1}^{4} \mathcal{E}_{i}(\rho) \\
& =\frac{-1}{\ln \rho} \int_{\partial \omega_{\rho}}\left(\nu \partial_{n} H-P_{H} \mathbf{n}\right) \cdot v_{0}(0) \mathrm{d} s+\sum_{i=1}^{5} \mathcal{E}_{i}(\rho) \\
& =\frac{-4 \pi \nu}{\ln \rho} \int_{\partial \omega_{\rho}}\left[\nu \partial_{n}\left(E u_{0}(0)\right)-\left(\Pi \cdot u_{0}(0)\right) \mathbf{n}\right] \cdot v_{0}(0) \mathrm{d} s+\sum_{i=1}^{5} \mathcal{E}_{i}(\rho)
\end{aligned}
$$

Then, the fact that $(E, \Pi)$ is the fundamental solution of the Stokes equations brings

$$
V_{F}(\rho)=-\frac{4 \pi \nu}{\ln \rho} u_{0}(0) \cdot v_{0}(0) \mathrm{d} s+\sum_{i=1}^{5} \mathcal{E}_{i}(\rho)
$$

Yet, we show that $\left|\mathcal{E}_{i}(\rho)\right|=o\left(\frac{-1}{\ln \rho}\right)$ for all $i=1, \ldots, 5$ (see the proofs in Sect. 4). Equation (3.1) is satisfied with

$$
f(\rho)=\frac{-1}{\ln \rho} \quad \text { and } \quad \delta_{F}=4 \pi \nu u_{0}(0) \cdot v_{0}(0) .
$$

\subsection{A theorem gathering the main results}

The following theorem provides the topological asymptotic expansion for the cost functionals under consideration. It results from Proposition 3.1 with the values of $f(\rho)$ and $\delta_{F}$ obtained before and the values of $\delta_{J}$ whose calculus is performed in Section 4 . 
TABLE 1 . Values of $\delta_{J}$ for the 3 categories of cost functional.

\begin{tabular}{|c|c|c|}
\hline & $3 \mathrm{D}$ & $2 \mathrm{D}$ \\
\hline \hline case 1 & 0 & 0 \\
\hline case 2 & 0 & 0 \\
\hline case 3 & $\mathcal{P}_{\omega} u_{0}(0) \cdot u_{0}(0)$ & $4 \pi \nu\left|u_{0}(0)\right|^{2}$ \\
\hline
\end{tabular}

Theorem 3.1. Under the hypotheses of Section 2, we have the asymptotic expansions

$$
\begin{gathered}
j(\rho)-j(0)=\rho\left[\mathcal{P}_{\omega} u_{0}(0) \cdot v_{0}(0)+\delta_{J}\right]+o(\rho) \quad \text { in } 3 D, \\
j(\rho)-j(0)=\frac{-1}{\ln \rho}\left[4 \pi \nu u_{0}(0) \cdot v_{0}(0)+\delta_{J}\right]+o\left(\frac{-1}{\ln \rho}\right) \quad \text { in } 2 D .
\end{gathered}
$$

The polarization matrix $\mathcal{P}_{\omega}$ is defined by (3.9), for a spherical hole it reads

$$
\mathcal{P}_{B(0,1)}=6 \pi \nu I
$$

The values of the term $\delta_{J}$ for the cost functionals of interest are indicated in Table 1 .

\section{Proofs}

\subsection{Preliminary lemmas}

The proofs of the first three lemmas can be found in [8] (Vol. 2 Sect. VIII.1 for Lems. 4.1 and 4.2 , Vol. 1 Sect. III.3 for Lem. 4.3).

Lemma $4.1(n=2,3)$. For all $(u, v) \in H_{0}^{1}\left(\Omega_{\rho}\right)^{n} \times H^{1}\left(\Omega_{\rho}\right)^{n}$ with div $v=0$,

$$
\int_{\Omega_{\rho}}(\nabla u \cdot v) \cdot u \mathrm{~d} x=0
$$

Lemma $4.2(n=2,3)$. For all $(u, v, w) \in H^{1}\left(\Omega_{\rho}\right)^{n} \times H^{1}\left(\Omega_{\rho}\right)^{n} \times H^{1}\left(\Omega_{\rho}\right)^{n}$,

$$
\left|\int_{\Omega_{\rho}}(\nabla u . v) \cdot w \mathrm{~d} x\right| \leq c(n, \Omega)|u|_{1, \Omega_{\rho}}\|v\|_{1, \Omega_{\rho}}\|w\|_{1, \Omega_{\rho}} .
$$

Moreover, if $v_{\mid \Gamma}=w_{\mid \Gamma}=0$,

$$
\left|\int_{\Omega_{\rho}}(\nabla u . v) . w \mathrm{~d} x\right| \leq k|u|_{1, \Omega_{\rho}}|v|_{1, \Omega_{\rho}}|w|_{1, \Omega_{\rho}},
$$

where $k$ is the constant defined by (2.6).

Lemma $4.3(n=2,3)$. Consider $R>0$ such that $B(0, R) \subset \Omega$. For any $w \in H^{1 / 2}(\Gamma)^{n}$ satisfying

$$
\int_{\Gamma} w \cdot \mathbf{n d} s=0
$$


there exists $W \in H^{1}(\Omega)^{n}$ such that

$$
\begin{array}{rlrl}
W_{\mid \Gamma} & =w, & \\
\operatorname{div} W & =0 \quad \text { in } \Omega, \\
W & =0 \quad \text { in } B(0, R), \\
|W|_{1, \Omega} & \leq c_{0}\|w\|_{\frac{1}{2}, \Gamma} .
\end{array}
$$

The constant $c_{0}$ may depend on $n, \Omega$ and $R$.

Lemma $4.4(n=2,3)$. Consider $f_{\rho} \in H^{-1}\left(\Omega_{\rho}\right)^{n}, V_{\rho} \in H^{1}\left(\Omega_{\rho}\right)^{n}$ with $\operatorname{div} V_{\rho}=0$ and $\left|V_{\rho}\right|_{1, \Omega_{\rho}} \leq \beta<\nu / k$ and $w \in H^{1 / 2}(\Gamma)^{n}$ verifying $\int_{\Gamma} w \cdot \mathbf{n d} s=0$. Let $\left(y_{\rho}, p_{y_{\rho}}\right) \in H^{1}\left(\Omega_{\rho}\right)^{n} \times L^{2}\left(\Omega_{\rho}\right)$ be a solution (if exists) of the problem:

$$
\left\{\begin{aligned}
-\nu \Delta y_{\rho}+\nabla y_{\rho} \cdot V_{\rho}+\nabla V_{\rho} \cdot y_{\rho}+\nabla y_{\rho} \cdot y_{\rho}+\nabla p_{y_{\rho}}=f_{\rho} & \text { in } \Omega_{\rho}, \\
\operatorname{div} y_{\rho}=0 & \text { in } \Omega_{\rho}, \\
y_{\rho}=w & \text { on } \Gamma, \\
y_{\rho}=0 & \text { on } \partial \omega_{\rho} .
\end{aligned}\right.
$$

There exists some constants $\gamma=\gamma(n, \Omega, \nu, \beta)$ and $c=c(n, \Omega, \nu, \beta)$ such that if $\|w\|_{\frac{1}{2}, \Gamma} \leq \gamma$, then

$$
\left\|y_{\rho}\right\|_{1, \Omega_{\rho}} \leq c\left[\left\|f_{\rho}\right\|_{-1, \Omega_{\rho}}+\|w\|_{\frac{1}{2}, \Gamma}\right] .
$$

Proof. Let us consider $W$ the extension of $w$ given by Lemma 4.3. The function $z_{\rho}=y_{\rho}-W$ satisfies

$$
\left\{\begin{aligned}
-\nu \Delta z_{\rho} & +\nabla z_{\rho} \cdot\left(V_{\rho}+W\right)+\nabla\left(V_{\rho}+W\right) \cdot z_{\rho}+\nabla z_{\rho} \cdot z_{\rho}+\nabla p_{z_{\rho}} \\
& =f_{\rho}+\nu \Delta W-\nabla W \cdot V_{\rho}-\nabla V_{\rho} \cdot W-\nabla W \cdot W \quad \text { in } \Omega_{\rho} \\
\operatorname{div} z_{\rho} & =0 \quad \text { in } \Omega_{\rho}, \\
z_{\rho}=0 & \text { on } \Gamma \\
z_{\rho}=0 & \text { on } \partial \omega_{\rho} .
\end{aligned}\right.
$$

Taking $z_{\rho}$ as test function in the variational formulation of the above problem brings

$$
\begin{aligned}
& \int_{\Omega_{\rho}}\left[\nu \nabla z_{\rho}: \nabla z_{\rho}+\left(\nabla z_{\rho} \cdot\left(V_{\rho}+W\right)+\nabla\left(V_{\rho}+W\right) \cdot z_{\rho}+\nabla z_{\rho} \cdot z_{\rho}\right) \cdot z_{\rho}\right] \mathrm{d} x= \\
& \qquad \int_{\Omega_{\rho}}\left[-\nu \nabla W: \nabla z_{\rho}+\left(f_{\rho}-\nabla W \cdot V_{\rho}-\nabla V_{\rho} . W-\nabla W \cdot W\right) . z_{\rho}\right] \mathrm{d} x .
\end{aligned}
$$

Thanks to Lemma 4.1, two terms vanish in the left hand side and we obtain

$$
\begin{aligned}
\nu \int_{\Omega_{\rho}} \nabla z_{\rho}: \nabla z_{\rho} \mathrm{d} x=-\int_{\Omega_{\rho}}\left(\nabla\left(V_{\rho}+W\right) \cdot z_{\rho}\right) \cdot z_{\rho} \mathrm{d} & \\
& +\int_{\Omega_{\rho}}\left[-\nu \nabla W: \nabla z_{\rho}+\left(f_{\rho}-\nabla W \cdot V_{\rho}-\nabla V_{\rho} \cdot W-\nabla W \cdot W\right) \cdot z_{\rho}\right] \mathrm{d} x .
\end{aligned}
$$

Lemma 4.2 and the Schwarz inequality yield

$$
\begin{aligned}
\nu\left|z_{\rho}\right|_{1, \Omega_{\rho}}^{2} \leq k\left|V_{\rho}\right|_{1, \Omega_{\rho}}\left|z_{\rho}\right|_{1, \Omega_{\rho}}^{2}+k|W|_{1, \Omega_{\rho}}\left|z_{\rho}\right|_{1, \Omega_{\rho}}^{2}+\nu|W|_{1, \Omega_{\rho}}\left|z_{\rho}\right|_{1, \Omega_{\rho}} & \\
& +\left\|f_{\rho}\right\|_{-1, \Omega_{\rho}}\left\|z_{\rho}\right\|_{1, \Omega_{\rho}}+c\|W\|_{1, \Omega_{\rho}}\left\|V_{\rho}\right\|_{1, \Omega_{\rho}}\left\|z_{\rho}\right\|_{1, \Omega_{\rho}}+c|W|_{1, \Omega_{\rho}}\|W\|_{1, \Omega_{\rho}}\left\|z_{\rho}\right\|_{1, \Omega_{\rho}},
\end{aligned}
$$


where $c$ denotes any positive constant depending only on $n, \Omega$ and $\nu$. Therefore, thanks to the Poincaré inequality and Lemma 4.3 , we have

$$
\left(\nu-k\left|V_{\rho}\right|_{1, \Omega_{\rho}}-k|W|_{1, \Omega_{\rho}}\right) c\left\|z_{\rho}\right\|_{1, \Omega_{\rho}} \leq c\|w\|_{\frac{1}{2}, \Gamma}+\left\|f_{\rho}\right\|_{-1, \Omega_{\rho}}+c\|w\|_{\frac{1}{2}, \Gamma}+c\|w\|_{\frac{1}{2}, \Gamma}^{2} .
$$

Yet, choosing $\gamma$ such that $c_{0} \gamma<\nu / k-\beta, c_{0}$ being the constant of Lemma 4.3 we have

$$
c_{1}:=\nu-k\left|V_{\rho}\right|_{1, \Omega_{\rho}}-k|W|_{1, \Omega_{\rho}} \geq \nu-k \beta-k c_{0} \gamma>0 .
$$

It follows that

$$
\left\|z_{\rho}\right\|_{1, \Omega_{\rho}} \leq \frac{1}{c_{1}}\left[c\|w\|_{\frac{1}{2}, \Gamma}+c\left\|f_{\rho}\right\|_{-1, \Omega_{\rho}}\right]
$$

Finally,

$$
\begin{aligned}
\left\|y_{\rho}\right\|_{1, \Omega_{\rho}} & \leq\left\|z_{\rho}\right\|_{1, \Omega_{\rho}}+\|W\|_{1, \Omega_{\rho}} \\
& \leq \frac{1}{c_{1}}\left[c\|w\|_{\frac{1}{2}, \Gamma}+c\left\|f_{\rho}\right\|_{-1, \Omega_{\rho}}\right]+c\|w\|_{\frac{1}{2}, \Gamma},
\end{aligned}
$$

which achieves the proof.

Lemma $4.5(n=3)$. Consider $\varphi \in H^{1 / 2}(\partial \omega)^{3}$ with $\int_{\partial \omega} \varphi \cdot \mathbf{n d} s=0$ and let $(v, p) \in W^{1}\left(\mathbb{R}^{3} \backslash \bar{\omega}\right)^{3} \times L^{2}\left(\mathbb{R}^{3} \backslash \bar{\omega}\right)$ be the solution of the problem:

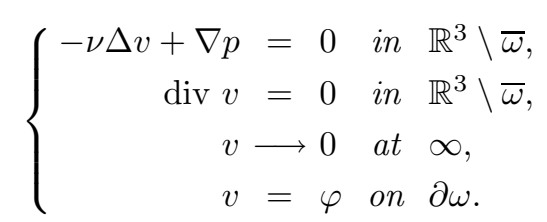

Then, for any $R>0$ and $D_{R}=\Omega \backslash \overline{B(0, R)}$, there exists some constant $c(R, \Omega)$ such that

$$
\begin{gathered}
\|v\|_{0, \Omega_{\rho} / \rho} \leq c \rho^{-1 / 2}\|\varphi\|_{1 / 2, \partial \omega} \\
|v|_{1, D_{R} / \rho} \leq c \rho^{1 / 2}\|\varphi\|_{1 / 2, \partial \omega} \\
|v|_{1, \Omega_{\rho} / \rho} \leq c\|\varphi\|_{1 / 2, \partial \omega} .
\end{gathered}
$$

Proof. See [11].

Lemma $4.6(n=2)$. Consider $\varphi \in H^{1 / 2}\left(\partial \omega_{\rho}\right)^{2}, \psi \in H^{1 / 2}(\Gamma)^{2}$ and let $\left(y_{\rho}, p_{y_{\rho}}\right) \in H^{1}\left(\Omega_{\rho}\right)^{2} \times L^{2}\left(\omega_{\rho}\right)$ be the solution of the problem:

$$
\left\{\begin{aligned}
-\nu \Delta y_{\rho}+\nabla p_{y_{\rho}}=0 & \text { in } \Omega_{\rho}, \\
\operatorname{div} y_{\rho}=0 & \text { in } \Omega_{\rho}, \\
y_{\rho}=\psi & \text { on } \Gamma \\
y_{\rho}=\varphi & \text { on } \partial \omega_{\rho} .
\end{aligned}\right.
$$

There exists some constant $c$ independent of $\rho$ such that

$$
\left\|y_{\rho}\right\|_{1, \Omega_{\rho}} \leq c\left[\|\psi\|_{\frac{1}{2}, \Gamma}+\frac{1}{\sqrt{-\ln \rho}}\|\varphi(\rho x)\|_{\frac{1}{2}, \partial \omega}\right] .
$$


Proof. If $\varphi$ is constant on $\partial \omega_{\rho}$ and $\psi=0$, we come down to a circular hole by using a principle of minimization of the energy and we use then the explicit expression of the solution (see [24]). Next, a decomposition of the solution and the standard elliptic regularity brings the case $\psi \neq 0$. Let us now study the case where $\varphi$ is not constant. Let $V$ be the bounded solution of

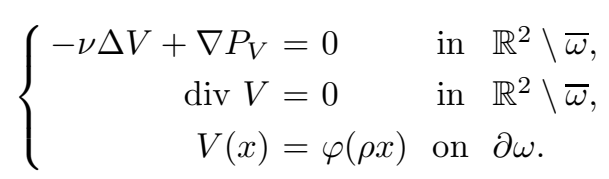

We have $V=\lambda+W$ with $\lambda \in \mathbb{R}$ and $W=O(1 / r)$. Then, we split $y_{\rho}$ into $W(x / \rho)+z_{\rho}$. The previous results apply to $z_{\rho}$ and $W(x / \rho)$ satisfies the desired estimate.

Lemma $4.7(n=2)$. Consider $f_{\rho} \in H^{-1}\left(\Omega_{\rho}\right)^{n}, V_{\rho} \in H^{1}\left(\Omega_{\rho}\right)^{n}$ with $\operatorname{div} V_{\rho}=0$ and $\left|V_{\rho}\right|_{1, \Omega_{\rho}} \leq \beta<\nu / k$, $\varphi \in H^{1 / 2}\left(\partial \omega_{\rho}\right)^{2}$ and $\psi \in H^{1 / 2}(\Gamma)^{2}$ verifying $\int_{\Gamma} \psi \cdot \mathbf{n d} s=0$. Let $\left(y_{\rho}, p_{y_{\rho}}\right) \in H^{1}\left(\Omega_{\rho}\right)^{n} \times L^{2}\left(\Omega_{\rho}\right)$ be a solution (if exists) of the problem:

$$
\left\{\begin{aligned}
-\nu \Delta y_{\rho}+\nabla y_{\rho} \cdot V_{\rho}+\nabla V_{\rho} \cdot y_{\rho}+\nabla y_{\rho} \cdot y_{\rho}+\nabla p_{y_{\rho}}=f_{\rho} & \text { in } \Omega_{\rho}, \\
\operatorname{div} y_{\rho}=0 & \text { in } \Omega_{\rho}, \\
y_{\rho}=\psi & \text { on } \Gamma, \\
y_{\rho}=\varphi & \text { on } \partial \omega_{\rho} .
\end{aligned}\right.
$$

There exists some constants $\gamma=\gamma(n, \Omega, \nu, \beta)$ and $c=c(n, \Omega, \nu, \beta)$ such that if $\|w\|_{\frac{1}{2}, \Gamma} \leq \gamma$, then

$$
\left\|y_{\rho}\right\|_{1, \Omega_{\rho}} \leq c\left[\left\|f_{\rho}\right\|_{-1, \Omega_{\rho}}+\frac{1}{\sqrt{-\ln \rho}}\|\varphi(\rho x)\|_{\frac{1}{2}, \partial \omega}+\|\psi\|_{\frac{1}{2}, \Gamma}\right] .
$$

Proof. It is a combination of Lemmas 4.6 and 4.4.

\subsection{Some regularity properties}

From the regularity assumptions made on the data, we get by using some regularity theorems for the NavierStokes equations (see [8] Sect. VIII.5) that:

- $\left(u_{0}, p_{u_{0}}\right) \in H^{2}(\Omega) \times H^{1}(\Omega)$;

- $\left(u_{0}, p_{u_{0}}\right) \in H^{\infty}(\mathcal{O}) \times H^{\infty}(\mathcal{O})$ for all open set $\mathcal{O}$ such that $\overline{\mathcal{O}} \subset \Omega$;

- $\left(v_{0}, p_{v_{0}}\right) \in H^{3}(\Omega) \times H^{2}(\Omega)$.

We are now in position to prove Theorem 3.1. We shall

(1) estimate all errors that have been introduced in the calculus of $\delta_{F}$ in Sections 3.2 and 3.3, so that equation (3.1) holds;

(2) check equation (3.2) for the cost functionals presented.

We will denote by $c$ any positive constant that may depend on $\Omega, \nu$ and $U$ but never on $\rho$. We consider some positive radius $R$ such that $\overline{B(0, R)} \subset \Omega$ and $D_{R}=\Omega \backslash \overline{B(0, R)}$.

\subsection{Calculus of $\delta_{F}$ in $3 \mathrm{D}$}

\subsubsection{Preliminary estimates}

(1) Estimate of $h_{\rho}$. Lemma 4.5, the continuity of $u_{0}$ (thanks to the Sobolev imbeddings) and a change of variable yield

$$
\begin{aligned}
\left\|h_{\rho}\right\|_{0, \Omega_{\rho}} & \leq c \rho, \\
\left|h_{\rho}\right|_{1, D_{R}} & \leq c \rho, \\
\left|h_{\rho}\right|_{1, \Omega_{\rho}} & \leq c \rho^{1 / 2} .
\end{aligned}
$$


(2) Estimate of $r_{\rho}$. As $\left|u_{0}\right|_{1, \Omega}<\nu / k$, we have for $\rho$ sufficiently small

$$
\left|u_{0}+h_{\rho}\right|_{1, \Omega_{\rho}} \leq \alpha<\nu / k .
$$

Thus, by Lemma 4.4,

$$
\begin{aligned}
\left\|r_{\rho}\right\|_{1, \Omega_{\rho}} & \leq c\left\|-\nabla h_{\rho} \cdot u_{0}-\nabla u_{0} \cdot h_{\rho}-\nabla h_{\rho} \cdot h_{\rho}\right\|_{-1, \Omega_{\rho}}+c\left\|h_{\rho}\right\|_{1 / 2, \Gamma} \\
& \leq c\left\|\nabla h_{\rho}\right\|_{-1, \Omega_{\rho}}+c\left\|h_{\rho}\right\|_{-1, \Omega_{\rho}}+c\left\|\nabla h_{\rho} \cdot h_{\rho}\right\|_{-1, \Omega_{\rho}}+c\left\|h_{\rho}\right\|_{1 / 2, \Gamma}
\end{aligned}
$$

since $u_{0} \in L^{\infty}(\Omega)$. Yet

$$
\left\|\nabla h_{\rho}\right\|_{-1, \Omega_{\rho}} \leq\left\|h_{\rho}\right\|_{0, \Omega_{\rho}}
$$

Moreover, using Lemma 4.2, we obtain immediately that

$$
\left\|\nabla h_{\rho} \cdot h_{\rho}\right\|_{-1, \Omega_{\rho}} \leq c\left|h_{\rho}\right|_{1, \Omega_{\rho}}\left\|h_{\rho}\right\|_{1, \Omega_{\rho}} .
$$

Therefore

$$
\begin{aligned}
\left\|r_{\rho}\right\|_{1, \Omega_{\rho}} & \leq c\left\|h_{\rho}\right\|_{0, \Omega_{\rho}}+c\left|h_{\rho}\right|_{1, \Omega_{\rho}}\left\|h_{\rho}\right\|_{1, \Omega_{\rho}}+c\left\|h_{\rho}\right\|_{1, D_{R}} \\
& \leq c \rho .
\end{aligned}
$$

(3) Estimate of $S_{\rho}$. Lemma 4.5 and a Taylor expansion of $u_{0}$ computed at the origin provide the inequalities

$$
\begin{aligned}
\left\|S_{\rho}\right\|_{0, \Omega_{\rho} / \rho} & \leq c \rho^{1 / 2}, \\
\left|S_{\rho}\right|_{1, D_{R} / \rho} & \leq c \rho^{3 / 2}, \\
\left|S_{\rho}\right|_{1, \Omega_{\rho} / \rho} & \leq c \rho .
\end{aligned}
$$

\subsubsection{Estimate of the errors $\mathcal{E}_{i}(\rho)$}

We will successively prove that $\left|\mathcal{E}_{i}(\rho)\right|=o(\rho)$ for $i=1, \ldots, 5$.

(1) An integration by parts taking into account the fact that div $\left(u_{\rho}-u_{0}\right)=0$ provides

$$
\mathcal{E}_{1}(\rho)=-\int_{\Omega}\left(\nabla v_{0} \cdot\left(u_{\rho}-u_{0}\right)\right) \cdot\left(u_{\rho}-u_{0}\right) \mathrm{d} x .
$$

Thus,

$$
\begin{aligned}
\left|\mathcal{E}_{1}(\rho)\right| & \leq\left\|\nabla v_{0}\right\|_{L^{\infty}(\Omega)}\left\|u_{\rho}-u_{0}\right\|_{L^{2}(\Omega)}^{2} \\
& \leq\left\|\nabla v_{0}\right\|_{L^{\infty}(\Omega)}\left(\left\|u_{\rho}-u_{0}\right\|_{L^{2}\left(\Omega_{\rho}\right)}^{2}+\left\|u_{0}\right\|_{L^{2}\left(\omega_{\rho}\right)}^{2}\right) \\
& \leq\left\|\nabla v_{0}\right\|_{L^{\infty}(\Omega)}\left[\left(\left\|h_{\rho}\right\|_{L^{2}\left(\Omega_{\rho}\right)}+\left\|r_{\rho}\right\|_{L^{2}\left(\Omega_{\rho}\right)}\right)^{2}+\left\|u_{0}\right\|_{L^{2}\left(\omega_{\rho}\right)}^{2}\right] \\
& \leq(c \rho+c \rho)^{2}+c \rho^{3} \\
& \leq c \rho^{2} .
\end{aligned}
$$

(2) Thanks to the regularity of $\left(u_{0}, p_{u_{0}}\right)$ and $\left(v_{0}, p_{v_{0}}\right)$ in the vicinity of the origin, we obtain immediately that

$$
\left|\mathcal{E}_{2}(\rho)\right| \leq c \rho^{2}
$$


(3) We have by a change of variable

$$
\mathcal{E}_{3}(\rho)=\rho \int_{\partial \omega}\left[\nu \partial_{n}\left(r_{\rho}(\rho x)\right)-\rho p_{r_{\rho}}(\rho x) \mathbf{n}\right] \cdot v_{0}(\rho x) \mathrm{d} s .
$$

Hence,

$$
\left|\mathcal{E}_{3}(\rho)\right| \leq c \rho\left\|\nu \partial_{n}\left(r_{\rho}(\rho x)\right)-\rho p_{r_{\rho}}(\rho x) \mathbf{n}\right\|_{-1 / 2, \partial \omega} .
$$

Let $B$ be some ball such that $\bar{\omega} \subset B$ and $\bar{B} \subset \Omega$. For all $\varphi \in H^{1}(B \backslash \bar{\omega})^{3}$ with $\operatorname{div} \varphi=0$ and $\varphi_{\mid \partial B}=0$ we have by the Green formula

$$
\begin{aligned}
-\nu \int_{\partial \omega}\left[\nu \partial_{n}\left(r_{\rho}(\rho x)\right)-\rho p_{r_{\rho}}(\rho x) \mathbf{n}\right] \cdot \varphi \mathrm{d} s= & \int_{B \backslash \bar{\omega}} \rho^{2}\left(\nu \Delta r_{\rho}(\rho x)-\nabla p_{r_{\rho}}(\rho x)\right) \cdot \varphi \mathrm{d} x+\int_{B \backslash \bar{\omega}} \rho \nu \nabla r_{\rho}(\rho x) \cdot \nabla \varphi \mathrm{d} x \\
= & \rho^{2} \int_{B \backslash \bar{\omega}}\left[\nabla r_{\rho} \cdot\left(u_{0}+h_{\rho}\right)+\nabla\left(u_{0}+h_{\rho}\right) \cdot r_{\rho}+\nabla r_{\rho} \cdot r_{\rho}+\nabla h_{\rho} \cdot u_{0}+\nabla u_{0} \cdot h_{\rho}\right. \\
& \left.+\nabla h_{\rho} \cdot h_{\rho}\right](\rho x) \cdot \varphi \mathrm{d} x+\rho \nu \int_{B \backslash \bar{\omega}} \nabla r_{\rho}(\rho x) \cdot \nabla \varphi \mathrm{d} x \\
= & \rho \int_{B \backslash \bar{\omega}}\left[\nabla R_{\rho} \cdot\left(U_{0}+H_{\rho}\right)+\nabla\left(U_{0}+H_{\rho}\right) \cdot R_{\rho}+\nabla R_{\rho} \cdot R_{\rho}+\nabla H_{\rho} \cdot U_{0}\right. \\
& \left.+\nabla U_{0} \cdot H_{\rho}+\nabla H_{\rho} \cdot H_{\rho}\right] \cdot \varphi \mathrm{d} x+\nu \int_{B \backslash \bar{\omega}} \nabla R_{\rho} \cdot \nabla \varphi \mathrm{d} x,
\end{aligned}
$$

where $R_{\rho}(x)=r_{\rho}(\rho x), H_{\rho}(x)=H_{\rho}(\rho x)$ and $U_{\rho}(x)=u_{\rho}(\rho x)$. Then, by Lemma 4.2 and the Poincaré inequality,

$$
\begin{aligned}
\left|\int_{\partial \omega}\left[\nu \partial_{n}\left(r_{\rho}(\rho x)\right)-\rho p_{r_{\rho}}(\rho x) \mathbf{n}\right] \cdot \varphi \mathrm{d} s\right| \leq c \rho\left[\left|R_{\rho}\right| .\left|U_{0}+H_{\rho}\right|+\left|U_{0}+H_{\rho}\right|\left|R_{\rho}\right|+\left|R_{\rho}\right|\left|R_{\rho}\right|\right. & +\left|H_{\rho}\right|\left|U_{0}+H_{\rho}\right| \\
& \left.+\left|U_{0}\right|\left\|H_{\rho}\right\|\right]\|\varphi\|+c\left|R_{\rho} \| \varphi\right|,
\end{aligned}
$$

where all the norms and semi-norms are taken in $H^{1}(B \backslash \bar{\omega})$. Next,

$$
\begin{aligned}
\left|\int_{\partial \omega}\left[\nu \partial_{n}\left(r_{\rho}(\rho x)\right)-\rho p_{r_{\rho}}(\rho x) \mathbf{n}\right] \cdot \varphi \mathrm{d} s\right| & \leq c \rho\left[\left|R_{\rho}\right|\left(\left|U_{0}+H_{\rho}\right|+\left|R_{\rho}\right|\right)+\left|H_{\rho}\right|\left|U_{0}+H_{\rho}\right|+\left|U_{0}\right|\left\|H_{\rho}\right\|+\rho^{-1}\left|R_{\rho}\right|\right]\|\varphi\| \\
& \leq c \rho\left[\left|R_{\rho}\right|\left(1+\left|H_{\rho}\right|+\left|R_{\rho}\right|\right)+\left|H_{\rho}\right|\left(1+\left|H_{\rho}\right|\right)+\left\|H_{\rho}\right\|+\rho^{-1}\left|R_{\rho}\right|\right]\|\varphi\|,
\end{aligned}
$$

since $U_{0}$ is of class $\mathcal{C}^{1}$ in $B \backslash \bar{\omega}$. A new change of variables and equations (4.1) and (4.2) yield

$$
\left|\int_{\partial \omega}\left[\nu \partial_{n}\left(r_{\rho}(\rho x)\right)-\rho p_{r_{\rho}}(\rho x) \mathbf{n}\right] \cdot \varphi \mathrm{d} s\right| \leq c \rho^{1 / 2}\|\varphi\|_{1 / 2, \partial \omega} .
$$

Finally,

(4) We have

$$
\left|\mathcal{E}_{3}(\rho)\right| \leq c \rho^{3 / 2} .
$$

$$
\begin{aligned}
\left|\mathcal{E}_{4}(\rho)\right| & \leq c \rho\left\|\nu \partial_{n} S_{\rho}-P_{S_{\rho}} \mathbf{n}\right\|_{-1 / 2, \partial \omega}\left\|v_{0}(\rho x)\right\|_{1 / 2, \partial \omega} \\
& \leq c \rho\left|S_{\rho}\right|_{1, B \backslash \bar{\omega}} \\
& \leq c \rho^{2} .
\end{aligned}
$$


(5) A Taylor expansion of $v_{0}$ yields straightforwardly

$$
\left|\mathcal{E}_{5}(\rho)\right| \leq c \rho^{2}
$$

\subsection{Calculus of $\delta_{F}$ in 2D}

\subsubsection{Preliminary estimates}

(1) Thanks to the implicit mapping theorem applied to the variational formulation of Problem (3.13) in $\rho=0$, we obtain easily that

$$
\lim _{\rho \rightarrow 0}\left\|H_{\rho}-H_{0}\right\|_{1, \Omega}=0
$$

from which it comes

$$
\left\|H_{\rho}\right\|_{1, \Omega} \leq c .
$$

Furthermore, the definition of $H$ provides

$$
\left\{\begin{array}{l}
\|H\|_{0, \Omega} \leq c \\
\|H\|_{1, D_{R}} \leq c \\
|H|_{1, \Omega_{\rho}} \leq c \sqrt{-\ln \rho} \\
\left.\|H\|_{W^{1, p}(\Omega)} \leq c \quad \forall p \in\right] 1,2[.
\end{array}\right.
$$

Hence we have the estimates

Next, $H_{\rho}$ verifies

$$
\left\{\begin{array}{l}
\left\|h_{\rho}\right\|_{0, \Omega_{\rho}} \leq \frac{c}{-\ln \rho} \\
\left\|h_{\rho}\right\|_{1, D_{R}} \leq \frac{c}{-\ln \rho} \\
\left|h_{\rho}\right|_{1, \Omega_{\rho}} \leq \frac{c}{\sqrt{-\ln \rho}}
\end{array}\right.
$$

with

$$
-\nu \Delta H_{\rho}+\nabla P_{H_{\rho}}=f_{\rho}
$$

$$
\begin{aligned}
f_{\rho}= & -\nabla H_{\rho} \cdot\left(u_{0}-\frac{1}{\ln \rho} H\right)-\left(\nabla u_{0}-\frac{1}{\ln \rho} \nabla H\right) \cdot H_{\rho}-\frac{1}{\ln \rho} \nabla H_{\rho} \cdot H_{\rho} \\
& -\nabla u_{0} \cdot H-\nabla H \cdot u_{0}-\frac{1}{\ln \rho} \nabla H . H .
\end{aligned}
$$

Using the Hölder inequality, we obtain that for any $q \in] 1,2[$,

$$
\left\|f_{\rho}\right\|_{L^{q}}(\Omega) \leq c
$$

Then, a regularity property (see [8]) yields

$$
\left\{\begin{aligned}
\left\|H_{\rho}\right\|_{W^{2, q}(\Omega)} & \leq c \\
\left\|P_{H_{\rho}}\right\|_{W^{1, q}(\Omega)} & \leq c
\end{aligned}\right.
$$

(2) Due to Lemma 4.7, we have

$$
\left\|r_{\rho}\right\|_{1, \Omega_{\rho}} \leq \frac{c}{\sqrt{-\ln \rho}}\left\|\left(-u_{0}-h_{\rho}\right)(\rho x)\right\|_{1 / 2, \partial \omega} .
$$


Yet,

$$
\begin{aligned}
\left(-u_{0}-h_{\rho}\right)(\rho x) & =-u_{0}(\rho x)-\frac{-1}{\ln \rho}\left[\left(\ln (\rho r) I-\mathbf{e}_{\mathbf{r}} \mathbf{e}_{\mathbf{r}}^{T}\right) u_{0}(0)-H_{\rho}(\rho x)\right] \\
& =-u_{0}(\rho x)+u_{0}(0)+\frac{1}{\ln \rho}\left[\left(\ln r I-\mathbf{e}_{\mathbf{r}} \mathbf{e}_{\mathbf{r}}{ }^{T}\right) u_{0}(0)-H_{\rho}(\rho x)\right] .
\end{aligned}
$$

Hence, using that $W^{2, q}(\Omega) \subset L^{\infty}(\Omega)$, we obtain

$$
\left\|\left(-u_{0}-h_{\rho}\right)(\rho x)\right\|_{1 / 2, \partial \omega} \leq c \rho+\frac{c}{-\ln \rho} \leq \frac{c}{-\ln \rho} .
$$

We arrive at the inequality

$$
\left\|r_{\rho}\right\|_{1, \Omega_{\rho}} \leq \frac{c}{(-\ln \rho)^{3 / 2}}
$$

4.4.2. Estimate of the errors $\mathcal{E}_{i}(\rho)$

We shall prove that $\left|\mathcal{E}_{i}(\rho)\right|=o(\rho)$ for all $i=1, \ldots, 5$.

(1) We obtain in the same way as in $3 \mathrm{D}$ that

$$
\left|\mathcal{E}_{1}(\rho)\right| \leq c\left(\frac{-1}{\ln \rho}\right)^{2} .
$$

(2) Like in $3 \mathrm{D}$, thanks to the regularity assumptions, we find that

$$
\left|\mathcal{E}_{2}(\rho)\right| \leq c \rho^{2} .
$$

(3) A change of variable furnishes

$$
\mathcal{E}_{3}(\rho)=\int_{\partial \omega}\left[\nu \partial_{n}\left(r_{\rho}(\rho x)\right)-\rho p_{r_{\rho}}(\rho x) \mathbf{n}\right] \cdot v_{0}(\rho x) \mathrm{d} s,
$$

from which it follows

$$
\left|\mathcal{E}_{3}(\rho)\right| \leq c\left\|\nu \partial_{n}\left(r_{\rho}(\rho x)\right)-\rho p_{r_{\rho}}(\rho x) \mathbf{n}\right\|_{-1 / 2, \partial \omega} .
$$

Let $B$ be again some ball such that $\bar{\omega} \subset B$ and $\bar{B} \subset \Omega$. For all $\varphi \in H^{1}(B \backslash \bar{\omega})^{2}$ with $\operatorname{div} \varphi=0$ and $\varphi_{\mid \partial B}=0$ we have by the Green formula

$$
\begin{aligned}
-\nu \int_{\partial \omega}\left[\nu \partial_{n}\left(r_{\rho}(\rho x)\right)-\rho p_{r_{\rho}}(\rho x) \mathbf{n}\right] \cdot \varphi \mathrm{d} s= & \int_{B \backslash \bar{\omega}} \rho^{2}\left(\nu \Delta r_{\rho}(\rho x)-\nabla p_{r_{\rho}}(\rho x)\right) \cdot \varphi \mathrm{d} x+\int_{B \backslash \bar{\omega}} \rho \nu \nabla r_{\rho}(\rho x) \cdot \nabla \varphi \mathrm{d} x \\
= & \rho^{2} \int_{B \backslash \bar{\omega}}\left[\nabla r_{\rho} \cdot\left(u_{0}+h_{\rho}\right)+\nabla\left(u_{0}+h_{\rho}\right) \cdot r_{\rho}+\nabla r_{\rho} \cdot r_{\rho}\right](\rho x) \cdot \varphi \mathrm{d} x \\
& +\rho \nu \int_{B \backslash \bar{\omega}} \nabla r_{\rho}(\rho x) \cdot \nabla \varphi \mathrm{d} x .
\end{aligned}
$$

A change of variable, Lemma 4.2 and the Poincaré inequality bring

$$
\begin{aligned}
\left|\int_{\partial \omega}\left[\nu \partial_{n}\left(r_{\rho}(\rho x)\right)-\rho p_{r_{\rho}}(\rho x) \mathbf{n}\right] \cdot \varphi \mathrm{d} s\right| \leq c \rho\left[\left|r_{\rho}(\rho x)\right| \cdot\left\|\left(u_{0}+h_{\rho}\right)(\rho x)\right\|+\mid\right. & \left(u_{0}+h_{\rho}\right)(\rho x) \mid\left\|r_{\rho}(\rho x)\right\| \\
& \left.+\left|r_{\rho}(\rho x)\right|\left\|r_{\rho}(\rho x)\right\|\right]\|\varphi\|+c\left|r_{\rho}(\rho x) \| \varphi\right|,
\end{aligned}
$$


where all the norms and semi-norms are taken in $H^{1}(B \backslash \bar{\omega})$. Next, easy manipulations yield

$$
\begin{aligned}
\left|\int_{\partial \omega}\left[\nu \partial_{n}\left(r_{\rho}(\rho x)\right)-\rho p_{r_{\rho}}(\rho x) \mathbf{n}\right] \cdot \varphi \mathrm{d} s\right| & \leq\left[c\left|r_{\rho}\right|_{1, \Omega_{\rho}}+c\left\|r_{\rho}\right\|_{1, \Omega_{\rho}}+c\left|r_{\rho}\right|_{1, \Omega_{\rho}}\left\|r_{\rho}\right\|_{1, \Omega_{\rho}}\right]\|\varphi\| \\
& \leq \frac{c}{(-\ln \rho)^{3 / 2}}\|\varphi\| .
\end{aligned}
$$

Therefore,

$$
\left|\mathcal{E}_{3}(\rho)\right| \leq \frac{c}{(-\ln \rho)^{3 / 2}} .
$$

(4) A change of variable and a Taylor expansion of $v_{0}$ computed at the origin yield

$$
\left|\mathcal{E}_{4}(\rho)\right| \leq c \rho\left\|\nu \partial_{n}\left(h_{\rho}(\rho x)\right)-\rho p_{h_{\rho}}(\rho x) \mathbf{n}\right\|_{-1 / 2, \partial \omega} .
$$

Then, arguing as for the estimation of $\mathcal{E}_{3}(\rho)$, we obtain that

$$
\left\|\nu \partial_{n}\left(h_{\rho}(\rho x)\right)-\rho p_{h_{\rho}}(\rho x) \mathbf{n}\right\|_{-1 / 2, \partial \omega} \leq c\left|h_{\rho}\right|_{1, \Omega_{\rho}} \leq \frac{c}{\sqrt{-\ln \rho}},
$$

from which we deduce

(5) We have

$$
\left|\mathcal{E}_{4}(\rho)\right| \leq \frac{c \rho}{\sqrt{-\ln \rho}}
$$

$$
\mathcal{E}_{5}(\rho)=\frac{-1}{\ln \rho} \int_{\omega_{\rho}}\left(\nu \Delta H_{\rho}-\nabla P_{H_{\rho}}\right) \mathrm{d} x .
$$

For a chosen $q \in] 1,2[$, the Hölder inequality yields

$$
\begin{aligned}
\left|\mathcal{E}_{5}(\rho)\right| & \leq \frac{-c}{\ln \rho}\left(\left\|H_{\rho}\right\|_{W^{2, q}(\Omega)}+\left\|P_{H_{\rho}}\right\|_{W^{1, q}(\Omega)}\right)\|1\|_{L^{q /(q-1)}\left(\omega_{\rho}\right)} \\
& \leq \frac{-c}{\ln \rho} \rho^{2-2 / q}
\end{aligned}
$$

\subsection{Calculus of $\delta_{J}$}

\subsubsection{Recall of some estimates}

In both Sections 3.2 and 3.3, we have split $u_{\rho}-u_{0}$ in the domain $\Omega_{\rho}$ into $u_{\rho}-u_{0}=h_{\rho}+r_{\rho}$ and we have proved that

$$
\left\{\begin{aligned}
\left\|h_{\rho}\right\|_{1, D_{R}} & =O(f(\rho)), \\
\left\|h_{\rho}\right\|_{0, \Omega_{\rho}} & =O(f(\rho)) \\
\left|h_{\rho}\right|_{1, \Omega_{\rho}} & =O(\sqrt{f(\rho)}), \\
\left\|r_{\rho}\right\|_{1, \Omega_{\rho}} & =O(f(\rho)),
\end{aligned}\right.
$$

with $f(\rho)=\rho$ in $3 \mathrm{D}, f(\rho)=-1 / \ln \rho$ in $2 \mathrm{D}$. It follows directly

$$
\left\{\begin{aligned}
\left\|u_{\rho}-u_{0}\right\|_{1, D_{R}} & =O(f(\rho)), \\
\left\|u_{\rho}-u_{0}\right\|_{0, \Omega_{\rho}} & =O(f(\rho)), \\
\left|u_{\rho}-u_{0}\right|_{1, \Omega_{\rho}} & =O(\sqrt{f(\rho)}) .
\end{aligned}\right.
$$

Let us now turn to the checking of equation (3.2) for the values of $\delta_{J}$ announced in Theorem 3.1. The three examples of functional are studied successively. 
(1) Case 1. This is an immediate consequence of the differentiability of $J$ and estimates (4.6).

(2) Case 2. We have

$$
\begin{aligned}
J_{\rho}\left(u_{\rho}\right)-J_{0}\left(u_{0}\right)-D J_{0}\left(u_{0}\right)\left(u_{\rho}-u_{0}\right) & =\left[J_{\rho}\left(u_{\rho}\right)-J_{0}\left(u_{\rho}\right)\right]+\left[J_{0}\left(u_{\rho}\right)-J_{0}\left(u_{0}\right)-D J_{0}\left(u_{0}\right)\left(u_{\rho}-u_{0}\right)\right] \\
& =-\int_{\omega_{\rho}}\left|u_{d}\right|^{2} \mathrm{~d} x+\int_{\Omega_{\rho}}\left|u_{\rho}-u_{0}\right|^{2} \mathrm{~d} x+\int_{\omega_{\rho}}\left|u_{0}\right|^{2} \mathrm{~d} x .
\end{aligned}
$$

On the one hand, from the regularity of $u_{d}$ and $u_{0}$, we derive

$$
\int_{\omega_{\rho}}\left|u_{d}\right|^{2} \mathrm{~d} x+\int_{\omega_{\rho}}\left|u_{0}\right|^{2} \mathrm{~d} x=o(f(\rho)) .
$$

On the other hand, estimates (4.6) imply

$$
\int_{\Omega}\left|u_{\rho}-u_{0}\right|^{2} \mathrm{~d} x=o(f(\rho)) .
$$

It follows that $\delta_{J}=0$.

(3) As in the previous case, the calculus gives

$$
\begin{aligned}
V_{J}(\rho) & :=J_{\rho}\left(u_{\rho}\right)-J_{0}\left(u_{0}\right)-D J_{0}\left(u_{0}\right)\left(u_{\rho}-u_{0}\right) \\
& =-\nu \int_{\omega_{\rho}}\left|\nabla u_{d}\right|^{2} \mathrm{~d} x+\nu \int_{\Omega_{\rho}}\left|\nabla\left(h_{\rho}+r_{\rho}\right)\right|^{2} \mathrm{~d} x+\nu \int_{\omega_{\rho}}\left|\nabla u_{0}\right|^{2} \mathrm{~d} x .
\end{aligned}
$$

Thanks to the regularity of $u_{0}$ and $u_{d}$ in the vicinity of the origin, the first and the third terms behave like a $o(f(\rho))$. We are now focusing on the second term.

(a) Let us first study the 3D case. It follows from estimates (4.1) and (4.2) that

$$
V_{J}(\rho)=\nu \int_{\Omega_{\rho}}\left|\nabla h_{\rho}\right|^{2} \mathrm{~d} x+o(f(\rho)) .
$$

The Green formula and a change of variable yield successively

$$
\begin{aligned}
V_{J}(\rho) & =-\int_{\partial \omega_{\rho}}\left(\nu \partial_{n} h_{\rho}-p_{h_{\rho}} \mathbf{n}\right) \cdot h_{\rho} \mathrm{d} s+o(f(\rho)) \\
& =-\rho \int_{\partial \omega}\left(\nu \partial_{n} H_{\rho}-P_{H_{\rho}} \mathbf{n}\right) \cdot H_{\rho} \mathrm{d} s+o(f(\rho)) .
\end{aligned}
$$

Using estimates (4.3), we obtain

$$
V_{J}(\rho)=-\rho \int_{\partial \omega}\left(\nu \partial_{n} H-P_{H} \mathbf{n}\right) \cdot H \mathrm{~d} s+o(f(\rho)) .
$$

Then, by the jump relation of the single layer potential,

$$
V_{J}(\rho)=-\rho \int_{\partial \omega} \eta \cdot u_{0}(0) \mathrm{d} s+o(f(\rho)),
$$

from which we deduce the announced expression of $\delta_{J}$. 


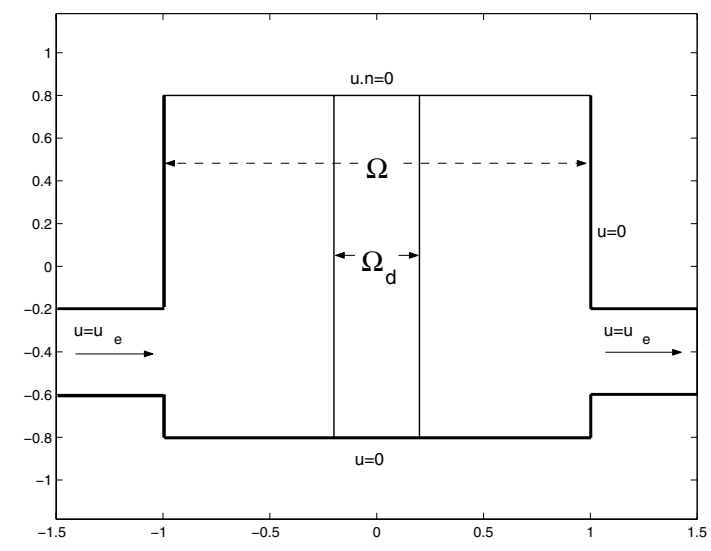

Figure 2. The domain and the boundary conditions.

(b) In 2D, the first step consists in applying the Green formula, which brings

$$
V_{J}(\rho)=-\int_{\partial \omega_{\rho}}\left[\nu \partial_{n}\left(h_{\rho}+r_{\rho}\right)-\left(p_{h_{\rho}}+p_{r_{\rho}}\right) \mathbf{n}\right] . u_{0} \mathrm{~d} s+o(f(\rho)) .
$$

Arguing as for the estimation of $\mathcal{E}_{3}(\rho), \mathcal{E}_{4}(\rho)$ and $\mathcal{E}_{5}(\rho)$, we obtain successively

$$
\begin{aligned}
V_{J}(\rho) & =-\int_{\partial \omega_{\rho}}\left(\nu \partial_{n} h_{\rho}-p_{h_{\rho}} \mathbf{n}\right) \cdot u_{0} \mathrm{~d} s+o(f(\rho)) \\
& =-\int_{\partial \omega_{\rho}}\left(\nu \partial_{n} h_{\rho}-p_{h_{\rho}} \mathbf{n}\right) \cdot u_{0}(0) \mathrm{d} s+o(f(\rho)) \\
& =-\frac{-1}{\ln \rho} \int_{\partial \omega_{\rho}}\left(\nu \partial_{n} H-P_{H} \mathbf{n}\right) \cdot u_{0}(0) \mathrm{d} s+o(f(\rho)) .
\end{aligned}
$$

Finally, using $(3.12)$ and the fact that $(E, \Pi)$ is the fundamental solution, this latter expression reads also

$$
V_{J}(\rho)=\frac{-1}{\ln \rho} 4 \pi \nu\left|u_{0}(0)\right|^{2}+o(f(\rho))
$$

which provides the expected value of $\delta_{J}$.

\section{A NUMERICAL EXAMPLE}

We illustrate the use of the topological asymptotic analysis on a shape optimization example which has been treated in [11] in the context of the Stokes equations (i.e. the viscosity is considered as infinite). The model represents a purification tank in which, for engineering reasons, some obstacles have to be inserted in order to approximate a target flow $u_{d}$. The goal is to find the best locations for these obstacles. The geometry (2D) and the boundary conditions are given in Figure 2. The initial velocity and pressure fields $u_{0}$ and $p_{u_{0}}$ satisfy the Navier-Stokes equations in $\Omega$ :

$$
\left\{\begin{aligned}
-\nu \Delta u_{0}+\nabla u_{0} \cdot u_{0}+\nabla p_{u_{0}} & =0 \\
\operatorname{div} u_{0} & =0
\end{aligned}\right.
$$



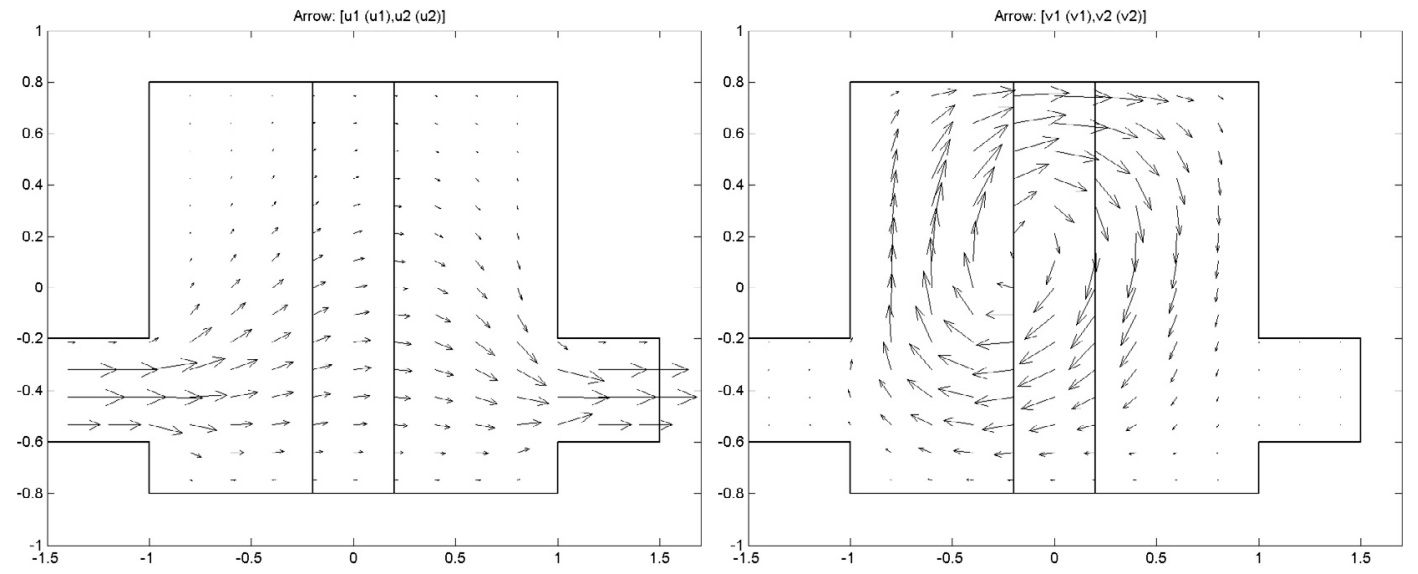

Figure 3. The direct and adjoint flows $(\mathrm{Re}=4)$.

The cost functional to be minimized is defined by

$$
\begin{aligned}
J(u) & =\int_{\Omega_{d}}\left|u-u_{d}\right|^{2} \mathrm{~d} x, \\
u_{d} & =\left(2 \times V_{\text {mean }} \times \frac{0.4(y+0.8)}{1.6^{2}}, 0\right),
\end{aligned}
$$

where $V_{\text {mean }}$ is the mean velocity of the fluid at the inlet. For that criterion, the topological asymptotic is given by Theorem 3.1 with $\delta_{J}=0$ : the topological gradient at the point $x_{0}$ reads

$$
g\left(x_{0}\right)=4 \pi \nu u_{0}\left(x_{0}\right) \cdot v_{0}\left(x_{0}\right)
$$

The obstacles are only allowed to be inserted in the right part of $\Omega \backslash \overline{\Omega_{d}}$. The topological optimization algorithm used here is the following.

- Initialization: choose $\Omega_{0}=\Omega$ and set $k=0$.

- Repeat until target is reached:

(1) solve the direct and adjoint problems in $\Omega_{k}$;

(2) compute the topological gradient $g$;

(3) seek $x^{*}=\operatorname{argmin}\left(g(x), x \in \Omega_{k}\right)$;

(4) set $\Omega_{k+1}=\Omega_{k} \backslash \overline{B\left(x^{*}, r_{0}\right)}$;

(5) $k \leftarrow k+1$.

The radius $r_{0}$ is fixed and chosen by the designer, here $r_{0}=0.03$. We present two experiments. For the first one, we have taken $V_{\text {mean }}=10$. This corresponds to a Reynolds number $R e=V_{\text {mean }} L / \nu, L$ being the inlet section, equal to 4 . The direct and adjoint flows and the topological gradient computed for the initial geometry are represented in Figures 3 and 4. Figure 5 illustrates the direct flow obtained after three iterations as well as the target flow. A convergence history of the cost functional is given in Figure 6. In the second configuration (Fig. 7), we have taken $V_{\text {mean }}=50(\mathrm{Re}=20)$. For higher Reynolds numbers, the appearance of turbulent structures makes inappropriate an optimization process based on the insertion of separated obstacles. 


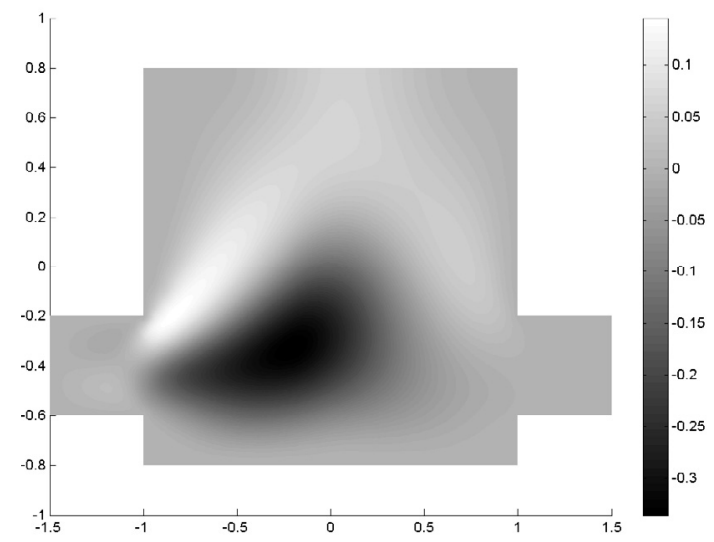

Figure 4. The topological gradient at the first iteration $(\mathrm{Re}=4)$.
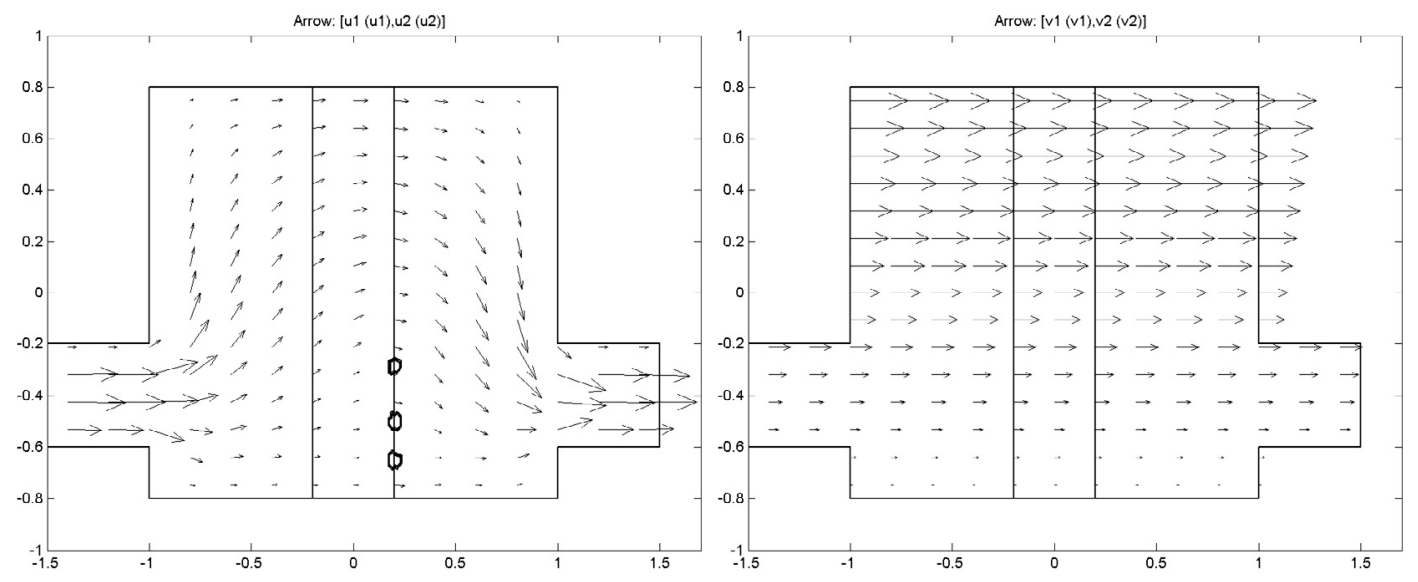

Figure 5 . The direct flow after 3 iterations and the target flow $(\operatorname{Re}=4)$.

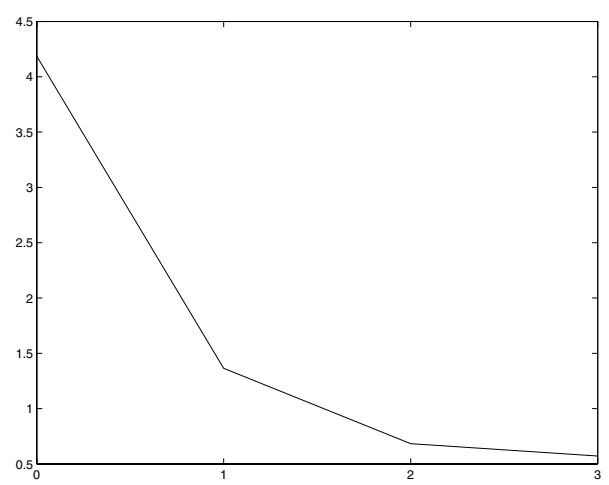

Figure 6. Convergence history $(\mathrm{Re}=4)$. 


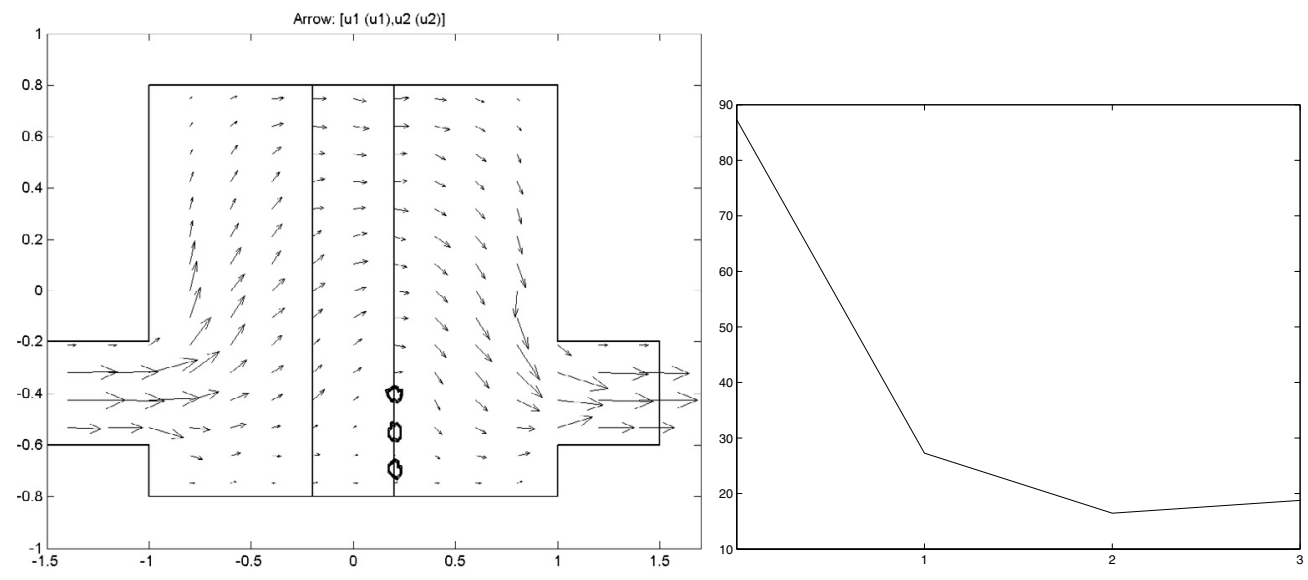

FiguRE 7 . The direct flow after 3 iterations and the convergence history $(\mathrm{Re}=20)$.

\section{REFERENCES}

[1] G. Allaire, Homogenization of the Navier-Stokes equations in open sets perforated with tiny holes. I. Abstract framework, a volume distribution of holes. Arch. Rational Mech. Anal. 113 (1990) 209-259.

[2] G. Allaire, Homogenization of the Navier-Stokes equations in open sets perforated with tiny holes. II. Noncritical sizes of the holes for a volume distribution and a surface distribution of holes. Arch. Rational Mech. Anal. 113 (1990) 261-298.

[3] G. Allaire, Shape optimization by the homogenization method. Springer, Appl. Math. Sci. 146 (2002).

[4] S. Amstutz, The topological asymptotic for the Helmholtz equation: insertion of a hole, a crack and a dielectric object. Rapport MIP No. 03-05 (2003).

[5] M. Bendsoe, Optimal topology design of continuum structure: an introduction. Technical report, Departement of mathematics, Technical University of Denmark, DK2800 Lyngby, Denmark (1996).

[6] R. Dautray and J.-L. Lions, Analyse mathématique et calcul numérique pour les sciences et les techniques. Masson, collection CEA 6 (1987).

[7] A. Friedman and M.S. Vogelius, Identification of small inhomogeneities of extreme conductivity byboundary measurements: a theorem of continuous dependence. Arch. Rational Mech. Anal. 105 (1989) 299-326.

[8] G. Galdi, An introduction to the mathematical theory of the Navier-Stokes equations. Vols. I and II, Springer-Verlag 39 (1994).

[9] S. Garreau, Ph. Guillaume and M. Masmoudi, The topological asymptotic for PDE systems: the elasticity case. SIAM J. Control Optim. 39 (2001) 1756-1778.

[10] Ph. Guillaume and K. Sid Idris, The topological asymptotic expansion for the Dirichlet problem. SIAM J. Control. Optim. 41 (2002) 1052-1072.

[11] Ph. Guillaume and K. Sid Idris, Topological sensitivity and shape optimization for the Stokes equations. Rapport MIP No. 01-24 (2001).

[12] M. Hassine and M. Masmoudi, The topological asymptotic expansion for the quasi-Stokes problem. ESAIM: COCV 10 (2004) $478-504$.

[13] A.M. Il'in, Matching of asymptotic expansions of solutions of boundary value problems. Translations Math. Monographs 102 (1992).

[14] J. Jacobsen, N. Olhoff and E. Ronholt, Generalized shape optimization of three-dimensionnal structures using materials with optimum microstructures. Technical report, Institute of Mechanical Engineering, Aalborg University, DK-9920 Aalborg, Denmark (1996).

[15] M. Masmoudi, The Toplogical Asymptotic, Computational Methods for Control Applications, R. Glowinski, H. Kawarada and J. Periaux Eds. GAKUTO Internat. Ser. Math. Sci. Appl. 16 (2001) 53-72.

[16] V. Mazya, S. Nazarov and B. Plamenevskij, Asymptotic theory of elliptic boundary value problems in singularly perturbed domains. Birkhäuser Verlag, Oper. Theory Adv. Appl. 101 (2000).

[17] S. Nazarov, A. Sequeira and J. Videman, Steady flows of Jeffrey-Hamel type from the half plane into an infinite channel. Linearization on an antisymmetric solution. J. Math. Pures Appl. 80 (2001) 1069-1098.

[18] S. Nazarov, A. Sequeira and J. Videman, Steady flows of Jeffrey-Hamel type from the half plane into an infinite channel. Linearization on a symmetric solution. J. Math. Pures Appl. 81 (2001) 781-810. 
[19] S. Nazarov and M. Specovius-Neugebauer, Approximation of exterior boundary value problems for the Stokes system. Asymptotic Anal. 14 (1997) 223-255.

[20] S. Nazarov, M. Specovius-Neugebauer and J. Videman, Nonlinear artificial boundary conditions for the Navier-Stokes equations in an aperture domain. Math. Nachr. 265 (2004) 24-67.

[21] B. Samet, S. Amstutz and M. Masmoudi, The topological asymptotic for the Helmholtz equation. SIAM J. Control Optim. 42 (2003) 1523-1544.

[22] B. Samet and J. Pommier, The topological asymptotic for the Helmholtz equation with Dirichlet condition on the boundary of an arbitrary shaped hole. SIAM J. Control Optim. 43 (2004) 899-921.

[23] A. Schumacher, Topologieoptimisierung von Bauteilstrukturen unter Verwendung von Lopchpositionierungkrieterien. Thesis, Universität-Gesamthochschule-Siegen (1995).

[24] K. Sid Idris, Sensibilité topologique en optimisation de forme. Thèse de l'INSA Toulouse (2001).

[25] J. Sokolowski and A. Zochowski, On the topological derivative in shape optimization. SIAM J. Control Optim. 37 (1999) 1241-1272.

[26] R. Temam, Navier-Stokes equations. Elsevier (1984). 